The Astrophysical JouRnal, 559:925-941, 2001 October 1

(C) 2001. The American Astronomical Society. All rights reserved. Printed in U.S.A.

\title{
A MODEL FOR ABUNDANCES IN METAL-POOR STARS
}

\author{
Y.-Z. QIAN ${ }^{1}$ AND G. J. WASSERBURG ${ }^{2}$ \\ Received 2001 February 12; accepted 2001 June 7
}

\begin{abstract}
A model is presented that seeks to explain quantitatively the stellar abundances of $r$-process elements and other elements associated with the $r$-process sites. It is argued that the abundances of all these elements in stars with $-3 \lesssim[\mathrm{Fe} / \mathrm{H}]<-1$ can be explained by the contributions of three sources. The sources are the first generations of very massive $\left(\gtrsim 100 M_{\odot}\right)$ stars that are formed from big bang debris and are distinct from Type II supernovae (SNe II) and two types of SNe II, the $H$ and $L$ events, which can occur only at $[\mathrm{Fe} / \mathrm{H}] \gtrsim-3$. The $H$ events are of high frequency and produce dominantly heavy $(A>130) r$-elements but no $\mathrm{Fe}$ (presumably leaving behind black holes). The $L$ events are of low frequency and produce $\mathrm{Fe}$ and dominantly light $(A \lesssim 130) r$-elements (essentially none above $\mathrm{Ba})$. By using the observed abundances in two ultra-metal-poor stars and the solar $r$-abundances, the initial or prompt inventory of elements produced by the first generations of very massive stars and the yields of $H$ and $L$ events can be determined. The abundances of a large number of elements in a star can then be calculated from the model by using only the observed $\mathrm{Eu}$ and $\mathrm{Fe}$ abundances. To match the model results and the observational data for stars with $-3<[\mathrm{Fe} / \mathrm{H}]<-1$ requires that the solar $r$-abundances for $\mathrm{Sr}, \mathrm{Y}, \mathrm{Zr}$, and $\mathrm{Ba}$ must be significantly increased from the standard values. No such changes appear to be required for all other elements. If the changes in the solar $r$-abundances for $\mathrm{Sr}, \mathrm{Y}, \mathrm{Zr}$, and $\mathrm{Ba}$ are not permitted, the model fails at $-3<[\mathrm{Fe} / \mathrm{H}]<-1$ but still works at $[\mathrm{Fe} / \mathrm{H}] \approx-3$ for these four elements. By using the corrected solar $r$-abundances for these elements, good agreement is obtained between the model results and data over the range $-3<[\mathrm{Fe} / \mathrm{H}]<-1$. No evidence of $s$-process contributions is found in this region, but all the observational data in this region now show regular increases of $\mathrm{Ba} / \mathrm{Eu}$ above the standard solar $r$-process value. Whether the solar $r$-components of $\mathrm{Sr}, \mathrm{Y}, \mathrm{Zr}$, and $\mathrm{Ba}$ used here to obtain a fit to the stellar data can be reconciled with those obtained from solar abundances by subtracting the $s$-components calculated from models is not clear.
\end{abstract}

Subject headings: Galaxy: evolution — stars: abundances - stars: Population II

\section{INTRODUCTION}

We present a phenomenological model that seeks to explain abundances of a large number of elements in ultrametal-poor $(\mathrm{UMP})$ stars with $[\mathrm{Fe} / \mathrm{H}] \approx-3$ and extend this model to metal-poor (MP) stars with $-3<[\mathrm{Fe} / \mathrm{H}]<-1$. Recently, Burris et al. (2000) reported abundances of heavy elements, both above and below $\mathrm{Ba}$, in 70 Galactic halo stars with a wide range in $[\mathrm{Fe} / \mathrm{H}]$. This new work and previous observations by, e.g., McWilliam et al. (1995) and Ryan, Norris, \& Beers (1996) stimulated us to address the problem of chemical evolution in the early Galaxy. Recent studies have discussed Galactic chemical evolution of $\mathrm{H}$ to Zn (Timmes, Woosley, \& Weaver 1995) and of heavy elements from $\mathrm{Ba}$ to Eu with both $s$-process and $r$-process contributions (Travaglio et al. 1999). These studies have shown general trends for abundance ratios of different elements to $\mathrm{Fe}$ as a function of $[\mathrm{Fe} / \mathrm{H}]$ that converge on solar values. Raiteri et al. (1999) treated temporal evolution of asymptotic giant branch (AGB) stars and rates of Type II supernovae (SNe II) and simulated Galactic Ba enrichment. Their results on $\mathrm{Ba} / \mathrm{Fe}$ show a wide scatter at low $[\mathrm{Fe} / \mathrm{H}]$ as observed. This was attributed to local inhomogeneities in the interstellar medium (ISM). In the present paper we will

\footnotetext{
${ }^{1}$ School of Physics and Astronomy, University of Minnesota, Minneapolis, MN 55455; qian@physics.umn.edu.

${ }^{2}$ Division of Geological and Planetary Sciences, California Institute of Technology, Pasadena, CA 91125.
}

show that the abundances in UMP and MP stars follow almost quantitatively from a set of simple rules.

The present study is purely phenomenological and confined to $-3 \lesssim[\mathrm{Fe} / \mathrm{H}]<-1$ where $\mathrm{SNe} \mathrm{Ia}$ (an Fe source) and low-mass AGB stars (the dominant s-process source) would not contribute significantly to the ISM. As pointed out by Truran (1981), abundances of neutron capture elements (e.g., $\mathrm{Ba}$ ) at low $[\mathrm{Fe} / \mathrm{H}]$ are dominated by $r$-process contributions from SNe II. We focus on the most extensive and self-consistent data set so far available, that of Burris et al. (2000), but treat data sets of Magain (1989), Gratton \& Sneden (1994), and Johnson \& Bolte (2001) as well. We will present the abundances resulting from production by the first generations of stars formed after the big bang and yields for two hypothesized types of SNe II. These results will be inferred from the observational data and not from theoretical models for the first generations of stars or for $r$-process production by SNe II. The $r$-process abundances ( $r$-abundances) calculated from our model for UMP and MP stars will be compared with the observational data. More specifically, the $r$-abundance of an element that represents the total abundance of all $r$-process isotopes of the element is calculated for comparison with observations as there are no data on isotopic $r$-abundances in stars other than the Sun. When referring to $r$-process nuclei, we consider both those that are the direct products of a rapid neutron capture scenario and those that may be produced by other processes closely related to the $r$-process in the same SN II event. The latter would include many nuclei 
with atomic masses of $A \sim 90$ that would be made in the $\alpha$-process (Woosley \& Hoffman 1992). If a sufficient neutron abundance existed at the end of the $\alpha$-process, the nuclei with $A \sim 90$ would become the seed nuclei to capture the neutrons during the subsequent $r$-process. Otherwise, these nuclei would experience no further processing and would be a part of the SN II ejecta. The $\alpha$-process could be the dominant source of $\mathrm{Sr}, \mathrm{Y}$, and $\mathrm{Zr}$.

\subsection{The Basis of the Phenomenological Model}

It is assumed here that $\mathrm{SNe}$ II are the source of $r$-process nuclei ( $r$-nuclei). The abundances of the $r$-nuclei ${ }^{182} \mathrm{Hf}$ and ${ }^{129} \mathrm{I}$ in the early solar system (ESS) are well established. These data require that the SNe II responsible for ${ }^{182} \mathrm{Hf}$ not produce any significant amounts of ${ }^{129} \mathrm{I}$ (Wasserburg, Busso, \& Gallino 1996, hereafter WBG96). The frequency of this type of SN II was argued to be $\sim\left(10^{7} \mathrm{yr}\right)^{-1}$ for a standard reference mass of hydrogen (see $\S 1.2$ ). The nuclide ${ }^{129}$ I was attributed to a different type of SN II with a frequency of $\sim\left(10^{8} \mathrm{yr}\right)^{-1}$. These hypothetical SN II types are called $H$ and $L$ events for "high" and "low" frequency, respectively. This approach assigns the dominant production of nuclei with $A>130$ ("heavy" $r$-nuclei) to the $H$ events and the nuclei with $A \lesssim 130$ ("light" $r$-nuclei) to the $L$ events. Qian, Vogel, \& Wasserburg (1998) developed a simple $r$-process model involving neutrinos to examine the different conditions in $H$ and $L$ events that are needed to provide a split between the production of heavy and light $r$-nuclei at $A \sim 130$. Because of their much higher frequency, the $H$ events were expected to be likely the first $\mathrm{SNe}$ II to inject $r$-nuclei into the ISM (WBG96). The effects of such "first-generation" SNe II should be apparent in the abundances of $r$-elements in UMP stars. Exquisite observations by Sneden et al. $(1996,2000)$ and Westin et al. (2000) have established that abundance ratios of many other heavy $r$-elements relative to Eu in UMP stars are remarkably constant and close to solar $r$-process values. This demonstrates that abundances of heavy $r$-elements in UMP stars appear to reflect production by pure $H$ events. Sneden et al. (2000) also found that the abundances of light $r$-elements such as Pd, Ag, and Cd in the UMP star CS 22892-052 were low relative to the solar $r$-abundance pattern that is translated to pass through the Eu data. This provides additional evidence that there should be at least two distinct types of SN II sources for the $r$-nuclei.

The available data on UMP stars (e.g., McWilliam et al. 1995; McWilliam 1998; Westin et al. 2000; Sneden et al. 2000; Burris et al. 2000) show that there is a wide range in the abundances of the heavy $r$-elements Eu and $\mathrm{Ba}(\sim 2 \mathrm{dex})$ for stars with $-3 \lesssim[\mathrm{Fe} / \mathrm{H}]<-2.5$. This clearly indicates that heavy $r$-elements, including the chronometer $\mathrm{Th}$, are produced without any significant coproduction of $\mathrm{Fe}$, thus requiring that heavy $r$-elements and $\mathrm{Fe}$ be produced in different types of SNe II. There is little Ba in stars with $-4 \lesssim[\mathrm{Fe} / \mathrm{H}]<-3$ (McWilliam et al. 1995; McWilliam 1998). Wasserburg \& Qian (2000, hereafter WQ00) inferred that the large dispersion in $\mathrm{Eu}$ and $\mathrm{Ba}$ abundances at $[\mathrm{Fe} / \mathrm{H}] \sim-3$ was caused by $H$ events adding heavy $r-$ elements but no $\mathrm{Fe}$ to a preexisting inventory of elements in the ISM. This preexisting inventory of $\mathrm{Fe}$ and associated elements was called the initial or prompt (hereafter $P$ ) inventory and attributed to the first generations of very massive $\left(\gtrsim 100 M_{\odot}\right)$ stars formed from big bang debris. It was further inferred that normal stars with masses of
1-60 $M_{\odot}$ could only form in a medium with sufficient metals to permit cooling during the collapse of a gas cloud. Normal stars with masses of $\sim 10-60 M_{\odot}$ would later become SNe II. Further evidence for the decoupling of heavy $r$-elements from $\mathrm{Fe}$ has been provided by the observations of the U-rich star CS 31082-001 (Cayrel et al. 2001). This star with $[\mathrm{Fe} / \mathrm{H}]=-2.9$ has extremely abundant $\mathrm{Os}$, Ir, Th, and U, sharply exhibiting the effect that little Fe is coproduced with the heavy $r$-elements as argued by Qian \& Wasserburg (2001b, hereafter QW01). Figure 1 shows the available $\mathrm{Ba}$ data over $-4 \lesssim[\mathrm{Fe} / \mathrm{H}] \lesssim-1$ and includes the Ba abundance inferred by QW01 for CS 31082-001 based on the three-component $(P, H$, and $L)$ model for abundances in UMP and MP stars. This inferred abundance (upper cross) is within $\approx 0.3$ dex of the preliminary Ba data on CS 31082-001 (lower cross) reported by Hill et al. (2001). As discussed above, the large scatter in Ba abundance at $-3 \lesssim[\mathrm{Fe} / \mathrm{H}]<-2.5$ shown in Figure 1 is attrib-

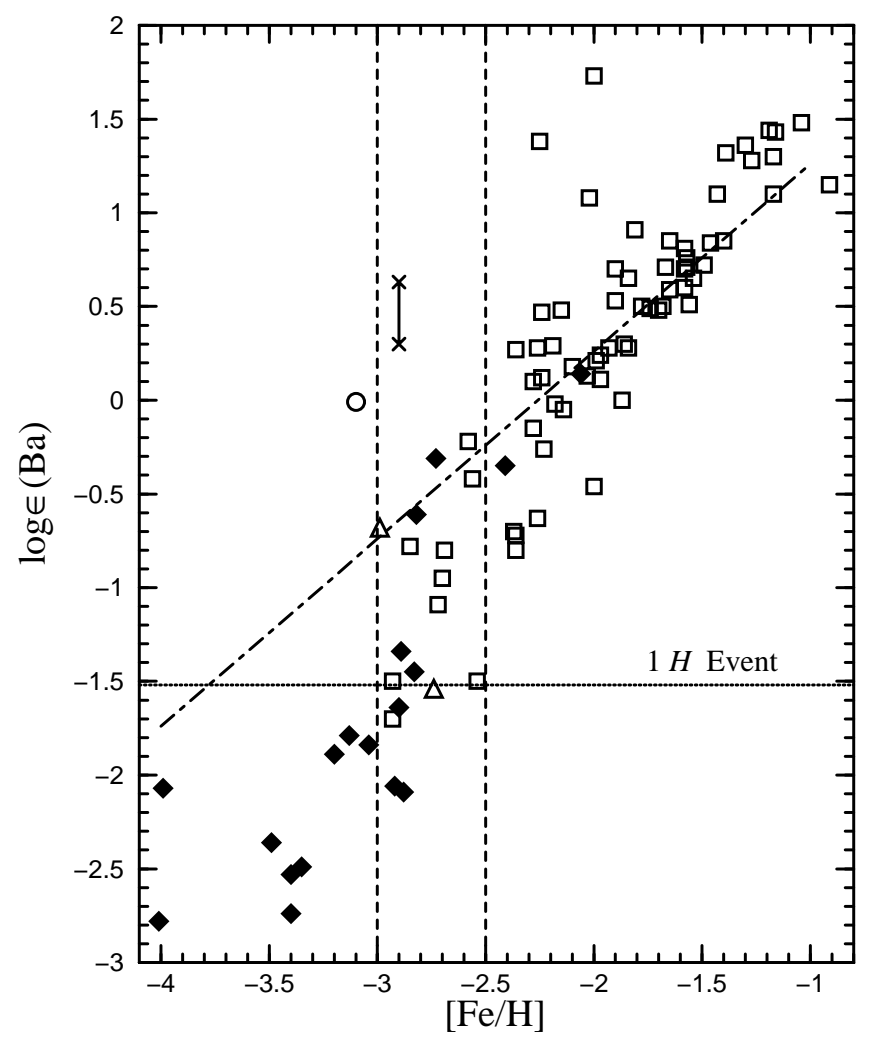

FIG. 1.-Data on $\log \epsilon(\mathrm{Ba})$ ( filled diamonds: McWilliam et al. 1995; McWilliam 1998; open triangles: Westin et al. 2000; open squares: Burris et al. 2000; open circle: Sneden et al. 2000) shown as a function of $[\mathrm{Fe} / \mathrm{H}]$. The upper cross indicates the Ba abundance inferred for CS 31082-001 by QW01 based on the three-component model and is within $\approx 0.3$ dex of the preliminary Ba data on this star (lower cross) reported by Hill et al. (2001). The dotted line labeled " $1 H$ Event" indicates the Ba yield of a single $H$ event. The low $\mathrm{Ba}$ abundances at $-4 \lesssim[\mathrm{Fe} / \mathrm{H}]<-3$ compared with this $H$ yield are attributed to very small admixtures of $H$ contributions into clouds having almost pure $P$-inventory composition. The rapid rise of $\log \epsilon(\mathrm{Ba})$ at $-3 \lesssim[\mathrm{Fe} / \mathrm{H}]<-2.5$ (between the two vertical dashed lines) is due to the onset of $H$ events. The dot-dashed line shows the mean trend of data at $[\mathrm{Fe} / \mathrm{H}]>-2.5$, where both $H$ and $L$ events occur, but cannot describe the data at lower $[\mathrm{Fe} / \mathrm{H}]$. Observed $\log \epsilon(\mathrm{Ba})$ values at $-3 \lesssim[\mathrm{Fe} / \mathrm{H}]<-2.5$ that correspond to $n_{H} \gtrsim 30 \mathrm{H}$ events (e.g., the open circle for CS 22892-052 and the linked crosses for CS 31082-001), as well as those at $[\mathrm{Fe} / \mathrm{H}]>-2.5$ that are far above the trend line, may represent enrichments by surface contamination in binaries instead of gross increases of $\mathrm{Ba}$ abundance in the ISM from which the stars formed. 
uted to the rapid occurrence of $H$ events shortly after the onset of normal star formation.

The scenario adopted here for the evolution of $r$-process related elements over Galactic history is schematically shown in Table 1 . Some time after the big bang, baryonic matter condenses in dark matter potential wells. Only very massive stars can form from big bang debris. These stars explode and contribute newly synthesized material. The number of such events is not known, but many such disrupting events may be required to give $[\mathrm{Fe} / \mathrm{H}] \approx-3$ in an average parcel of the ISM or intergalactic medium (IGM). The ISM or IGM now has a changed chemical composition compared with big bang debris. When some of this material cools, there is condensation of gas to form a more or less normal stellar population that includes the progenitors for SN II $H$ and $L$ events and lower mass stars. This state of condensation can be achieved when the abundance of all metals in the ISM or IGM reaches or surpasses some critical value. A nominal value of $[\mathrm{Fe} / \mathrm{H}] \approx-3$ for the critical "metallicity" was inferred by WQ00 from the observational data on UMP stars. This appears to be supported by the recent results of Bromm et al. (2001) who have simulated the collapse and fragmentation of gas clouds with different metallicities. The nominal value $[\mathrm{Fe} / \mathrm{H}] \approx-3$ will be used below for the $P$-inventory of $\mathrm{Fe}$ although the onset of "normal" astration may correspond to a possible range of $-4<[\mathrm{Fe} / \mathrm{H}]<-2.7$. The first SNe II to contribute $r$ elements to the ISM are the most frequent and most probable $H$ events. On a timescale of $>10^{8} \mathrm{yr}$, the relative contributions of $H$ and $L$ events should approach welldefined values that are determined by the average frequency ratio of $\sim 10: 1$ and the yields of these events. From then on the evolution of $r$-elements and $\mathrm{Fe}$ would progress as if there were only one type of SN II with small statistical deviations (see the evolution of $\mathrm{Ba}$ abundance relative to $\mathrm{Fe}$ at $[\mathrm{Fe} / \mathrm{H}]>-2.5$ indicated by the dot-dashed line in Fig. 1). At $[\mathrm{Fe} / \mathrm{H}] \sim-1, \mathrm{SNe}$ Ia begin to contribute and dominate the $\mathrm{Fe}$ production, as inferred from the $\mathrm{O}$ data (e.g., Timmes et al. 1995; Qian \& Wasserburg 2001a).

This approach sharply separates heavy-element production in the earliest epochs (epochs 2 and 3 in Table 1) from later production by SNe II (epochs 6 and 7) and from even later production by $\mathrm{SNe}$ Ia and contributions of evolved normal low-mass stars. The numbers of $H$ and especially $L$ events through epochs 6 and 7 are not very large (see $\S 1.3$ ) so that a discrete model is required.

For simplicity it is assumed that each $H$ or $L$ event has a fixed relative abundance pattern for the elements produced in each case and that the yields are constant for each $H$ or $L$ event. The average frequencies of these events are also assumed to be constant for a standard reference mass of hydrogen. The main results of the model only depend on the assumption of fixed yield patterns for $H$ and $L$ events (see $\S \S 1.2$ and 1.3). The key parameters of the model that must be determined by consideration of the observational data are the yields of $H$ and $L$ events for pertinent elements and the $P$-inventory composition that defines the baseline to which the contributions of $H$ and $L$ events are added. As we are concerned with the addition of elements to the ISM or IGM whose hydrogen mass fraction is essentially not altered during Galactic chemical evolution, we will typically use a representation where the abundances are given relative to hydrogen. This is the standard spectroscopic notation $\log \epsilon(\mathrm{E}) \equiv \log (\mathrm{E} / \mathrm{H})+12$ for element $\mathrm{E}$ where $\mathrm{E}$ is also used to represent the number of $\mathrm{E}$ atoms. The common notation $[\mathrm{E} / \mathrm{Fe}]$ complicates the discussion and is avoided here as ranges in $[\mathrm{E} / \mathrm{Fe}]$ may result from addition of either element $\mathrm{E}$ or $\mathrm{Fe}$.

\subsection{Time-Dependent Evolution and Mixing}

Consider a homogeneous system of gas with a timedependent total number $\mathrm{H}$ of hydrogen atoms. The rate of change in the total number $\mathrm{E}$ of $\mathrm{E}$ atoms in this gas may be written as

$$
\frac{d \mathrm{E}}{d t}=\sum_{i} P_{\mathrm{E}, i}+\left(\frac{\mathrm{E}}{\mathrm{H}}\right) \frac{d \mathrm{H}}{d t},
$$

where $\sum_{i} P_{\mathrm{E}, i}$ is the total rate for injection of element $\mathrm{E}$ into the gas after production by its sources and the last term accounts for the removal of matter from the gas by either astration or fragmentation ( $d \mathrm{H} / d t<0$ for both cases). The above equation may be rewritten as

$$
\frac{d(\mathrm{E} / \mathrm{H})}{d t}=\frac{\sum_{i} P_{\mathrm{E}, i}}{\mathrm{H}} .
$$

Thus, if SN II $H$ and $L$ events are the only sources under consideration for element $\mathrm{E}$ and the $\mathrm{SN}$ II frequencies are proportional to the amount of hydrogen in the gas, then equation (2) reduces to the following for a homogeneous mass of gas:

$$
\left(\frac{\mathrm{E}}{\mathrm{H}}\right)=\left(\frac{\mathrm{E}}{\mathrm{H}}\right)_{P}+n_{H}\left(\frac{\mathrm{E}}{\mathrm{H}}\right)_{H}+n_{L}\left(\frac{\mathrm{E}}{\mathrm{H}}\right)_{L},
$$

or in the spectroscopic notation,

$$
10^{\log \epsilon(\mathrm{E})}=10^{\log \epsilon(\mathrm{E})}+n_{H} \times 10^{\log \epsilon_{H}(\mathrm{E})}+n_{L} \times 10^{\log \epsilon_{L}(\mathrm{E})} .
$$

In equation (3), $(\mathrm{E} / \mathrm{H})_{P}$ is the $P$-inventory, $(\mathrm{E} / \mathrm{H})_{H}$ or $(\mathrm{E} / \mathrm{H})_{L}$ is the number of $\mathrm{E}$ atoms produced per hydrogen atom in the gas phase by each $H$ or $L$ event for a standard reference mass of hydrogen that is assumed here to mix with the ejecta in each case, and $n_{H}$ or $n_{L}$ is the number of $H$ or $L$ events that have occurred in this reference mass.

Now consider the mixture of two different gas systems (with subscripts " 1 " and " 2 ") that have different nucleosynthetic histories. For fixed values of $(\mathrm{E} / \mathrm{H})_{P},(\mathrm{E} / \mathrm{H})_{H}$, and $(\mathrm{E} / \mathrm{H})_{L}$, the abundance of element $\mathrm{E}$ in the mixture of two gas masses can be written as

$$
\begin{aligned}
\left(\frac{\mathrm{E}}{\mathrm{H}}\right)_{\text {mix }} & =\frac{(\mathrm{E} / \mathrm{H})_{1}(\mathrm{H})_{1}+(\mathrm{E} / \mathrm{H})_{2}(\mathrm{H})_{2}}{(\mathrm{H})_{1}+(\mathrm{H})_{2}} \\
& =\left(\frac{\mathrm{E}}{\mathrm{H}}\right)_{P}+\tilde{n}_{H}\left(\frac{\mathrm{E}}{\mathrm{H}}\right)_{H}+\tilde{n}_{L}\left(\frac{\mathrm{E}}{\mathrm{H}}\right)_{L},
\end{aligned}
$$

where

$$
\begin{gathered}
\tilde{n}_{H}=\frac{\left(n_{H}\right)_{1}(\mathrm{H})_{1}+\left(n_{H}\right)_{2}(\mathrm{H})_{2}}{(\mathrm{H})_{1}+(\mathrm{H})_{2}} \\
\tilde{n}_{L}=\frac{\left(n_{L}\right)_{1}(\mathrm{H})_{1}+\left(n_{L}\right)_{2}(\mathrm{H})_{2}}{(\mathrm{H})_{1}+(\mathrm{H})_{2}}
\end{gathered}
$$


TABLE 1

Chronology of Evolution

\begin{tabular}{|c|c|}
\hline Epoch & Description \\
\hline & Big bang \\
\hline $2 \ldots \ldots$ & $\begin{array}{l}\text { Formation and explosion of very massive }\left(\gtrsim 100 M_{\odot}\right) \text { stars in regions of baryonic matter condensation with production of "metals" } \\
(\mathrm{C}, \mathrm{N}, \mathrm{O}, \mathrm{Mg}, \mathrm{Al}, \mathrm{Si}, \ldots, \mathrm{Fe}, \ldots, \mathrm{Sr}, \mathrm{Y}, \mathrm{Zr})\end{array}$ \\
\hline $3 \ldots \ldots$ & $\begin{array}{l}\text { Disruption of condensed regions by explosions of very massive stars, return of material with "metals" to the IGM, and reionization } \\
\text { of the IGM }\end{array}$ \\
\hline $4 \ldots . .$. & Cooling and condensation of some of the baryonic matter in the IGM \\
\hline $5 \ldots . .$. & Formation of normal stellar populations with masses of $\sim 1-60 M_{\odot}$ when a metallicity of $-4<[\mathrm{Fe} / \mathrm{H}]<-2.7$ was reached \\
\hline $6 \ldots .$. & Occurrence of the first SN II $H$ events [with a frequency of $\sim\left(10^{7} \mathrm{yr}\right)^{-1}$ for a standard reference mass of hydrogen] \\
\hline $7 \ldots \ldots$ & Coeval occurrence of SN II $H$ and $L$ events [with an $L$-frequency of $\sim\left(10^{8} \mathrm{yr}\right)^{-1}$ for a standard reference mass of hydrogen] \\
\hline $8 \ldots \ldots$ & $\begin{array}{l}\text { Later occurrence of } \mathrm{SNe} \text { Ia with major Fe addition and evolution of low-mass AGB stars resulting in substantial } s \text {-process contributions } \\
\text { to the ISM at }[\mathrm{Fe} / \mathrm{H}] \gtrsim-1\end{array}$ \\
\hline
\end{tabular}

It can be seen that a mixture of two gas systems with different nucleosynthetic histories will result in a new system with effective values of $\tilde{n}_{H}$ and $\tilde{n}_{L}$ corresponding to the weighted averages of the contributing systems. As a result, all that mixing will do is change the values of $n_{H}$ and $n_{L}$ but still produce abundance patterns congruent to those resulting from $H$ and $L$ events. If the absolute yields of $H$ and $L$ events were to shift but the relative yields of one element to the other were maintained in each type of event, then the only result of this shift would again be to change $n_{H}$ and $n_{L}$, but the same congruence of abundance patterns would be maintained. It follows that a wide range of removal by astration and mixing of matter with different chemical evolution lead to a result that is indistinguishable from a simple history if the $P$-inventory composition and the basic relative yield templates of $H$ and $L$ events are fixed as assumed in this model. Thus, complex mixing and transport models are not required to pursue the approach used here but are effectively represented by the $n_{H}$ and $n_{L}$ values for a star. If the model assumptions are correct, then they provide a full basis for quantitatively determining the $r$-abundances in UMP and MP stars.

The frequencies of $H$ and $L$ events inferred from the abundances of ${ }^{182} \mathrm{Hf}$ and ${ }^{129} \mathrm{I}$ in the ESS, $\sim\left(10^{7} \mathrm{yr}\right)^{-1}$ and $\sim\left(10^{8} \mathrm{yr}\right)^{-1}$, respectively, correspond to a reference mass of hydrogen of $M_{\mathrm{H}}^{\text {ref }} \sim 3 \times 10^{4} M_{\odot}$ for a total SN II rate of $\sim(30 \mathrm{yr})^{-1}$ and a total gas mass of $\sim 10^{10} M_{\odot}$ in the present Galaxy (Qian \& Wasserburg 2001a). This reference mass is also the total amount of ISM typically swept by an SN II remnant (e.g., Thornton et al. 1998). We take this as the standard reference mass of hydrogen to mix with the nucleosynthetic products of an SN II $H$ or $L$ event. We will use equation (4) with fixed parameters $\log \epsilon_{P}(\mathrm{E}), \log \epsilon_{H}(\mathrm{E})$, and $\log \epsilon_{L}(\mathrm{E})$ to discuss abundances in UMP and MP stars. All quantities in this equation will be calculated for the above reference mass of hydrogen. The absolute yield of element $\mathrm{E}$ in units of mass for an $H$ or $L$ event, $Y_{H}(\mathrm{E})$ or $Y_{L}(\mathrm{E})$, can be estimated from the relationships

$$
\begin{aligned}
& \left(\frac{\mathrm{E}}{\mathrm{H}}\right)_{H}=\frac{Y_{H}(\mathrm{E}) /\left\langle A_{\mathrm{E}}\right\rangle_{H}}{M_{\mathrm{H}}^{\text {ref }}}, \\
& \left(\frac{\mathrm{E}}{\mathrm{H}}\right)_{L}=\frac{Y_{L}(\mathrm{E}) /\left\langle A_{\mathrm{E}}\right\rangle_{L}}{M_{\mathrm{H}}^{\text {ref }}},
\end{aligned}
$$

where $\left\langle A_{\mathrm{E}}\right\rangle_{H}$ or $\left\langle A_{\mathrm{E}}\right\rangle_{L}$ is the average atomic mass for the $\mathrm{E}$ isotopes produced in an $H$ or $L$ event.
In discussing the stellar observations, we consider that the observed stars formed from the immediate precursor ISM with the homogenized $r$-abundances of that local region. These $r$-abundances are not considered to have been altered by the subsequent evolution of the star. If the surface of a star is contaminated by some form of mass transfer from a companion (or nearby) SN II, then the observed abundance pattern should represent the products of that SN II (an $H$ or $L$ event), but the abundances would be greatly enhanced from those with which the star formed. For example, the extremely high Ba abundances in CS 31082-001 (crosses linked by a line) and CS 22892-052 (open circle) shown in Figure 1 may have resulted from surface contamination by the SN II explosion (of the $H$ type) of their respective binary companions (QW01). The issue of what level of $r$-process enrichment is so large that an SN II companion (or neighbor) appears to be required has been discussed by QW01 and will be considered below (see $\S 3.2$ ).

\subsection{Calculation of $n_{H}$ and $n_{L}$}

Given the $P$-inventory, $\log \epsilon_{P}(\mathrm{E})$, and the yields of $H$ and $L$ events, $\log \epsilon_{H}(\mathrm{E})$ and $\log \epsilon_{L}(\mathrm{E})$, the abundance of element $\mathrm{E}$ in a star, $\log \epsilon(\mathrm{E})$, can be calculated from equation (4). The numbers of contributing $H$ and $L$ events for the star, $n_{H}$ and $n_{L}$, are required for this calculation. If a certain element is exclusively produced by $H$ or $L$ events, then $n_{H}$ or $n_{L}$ can be simply obtained from the observed abundance of this element in the star by using the yield of an $H$ or $L$ event for this element.

Data on $\mathrm{Ba} \quad(A \sim 135)$ at $-4 \lesssim[\mathrm{Fe} / \mathrm{H}]<-3$ (McWilliam et al. 1995; McWilliam 1998) indicate that the $P$-inventory of heavy $r$-elements is negligible (WQ00; see Fig. 1). Observations also show that the abundances of $\mathrm{Ba}$ and above in UMP stars very closely follow the solar $r$ abundance pattern (Sneden et al. 1996, 2000; Westin et al. 2000). We consider that $H$ events are the exclusive source for the heavy $r$-elements above $\mathrm{Ba}$ (possibly including $\mathrm{Ba}$ ) with a yield pattern identical to the corresponding part of the solar $r$-abundance pattern (see $\S \S 2.1$ and 3.1). Then the $H$ yield of any heavy $r$-element above Ba can be used to calculate the value $n_{H}$ for a star. We use Eu $(A \sim 151)$ for this purpose as it is typically observed in UMP and MP stars and its solar inventory is dominated by $r$-process contributions. Specifically, the solar $r$-abundance of $\mathrm{Eu}$, $\log \epsilon_{\odot, r}(\mathrm{Eu})=0.52$ (Arlandini et al. 1999), was contributed by $n_{H}^{\odot}=10^{3} H$ events for a frequency of $f_{H}=\left(10^{7} \mathrm{yr}\right)^{-1}$ over a period of $10^{10} \mathrm{yr}$ prior to solar system formation 
$(\mathrm{SSF} ; \quad$ see $\quad \S \quad 1.4) . \quad$ Thus, $\quad \log \epsilon_{H}(\mathrm{Eu})=\log \epsilon_{\odot, r}(\mathrm{Eu})$ $-\log n_{H}^{\odot}=-2.48$. The value $n_{H}$ for a star is then

$$
n_{H}=10^{\log \epsilon(\mathrm{Eu})-\log \epsilon_{H}(\mathrm{Eu})}=10^{\log \epsilon(\mathrm{Eu})+2.48} .
$$

As little or no $\mathrm{Fe}$ is produced in $H$ events (see $\S 1.1$ ), we consider that the $\mathrm{Fe}$ abundance at $-3<[\mathrm{Fe} / \mathrm{H}]<-1$ results from contributions of $L$ events being added to the $P$-inventory:

$$
10^{\log \epsilon(\mathrm{Fe})}=10^{\log \epsilon_{P}(\mathrm{Fe})}+n_{L} \times 10^{\log \epsilon_{L}(\mathrm{Fe})} .
$$

As SNe Ia were the dominant $\mathrm{Fe}$ source at $[\mathrm{Fe} / \mathrm{H}]>-1$, only a fraction $\alpha_{\mathrm{Fe}}$ of the solar Fe inventory $\log \epsilon_{\odot}(\mathrm{Fe})=$ 7.51 (Anders \& Grevesse 1989) was contributed by SN II $L$ events. For $\alpha_{\mathrm{Fe}}=\frac{1}{3}$ (e.g., Timmes et al. 1995; Qian \& Wasserburg 2001a) and with $n_{L}^{\odot}=10^{2}$ contributing $L$ events for a frequency of $f_{L}=\left(10^{8} \mathrm{yr}\right)^{-1}$ over a period of $10^{10} \mathrm{yr}$ prior to $\mathrm{SSF}$ (see $\S 1.4), \log \epsilon_{L}(\mathrm{Fe})=\log \alpha_{\mathrm{Fe}}+\log \epsilon_{\odot}(\mathrm{Fe})$ $-\log n_{L}^{\odot}=5.03$ corresponding to $[\mathrm{Fe} / \mathrm{H}]_{L}=\log \epsilon_{L}(\mathrm{Fe})$ $-\log \epsilon_{\odot}(\mathrm{Fe})=-2.48$. We take $\log \epsilon_{P}(\mathrm{Fe}) \approx 4.51$ corresponding to $[\mathrm{Fe} / \mathrm{H}]_{P} \approx-3$. The $P$-inventory of $\mathrm{Fe}$ is overwhelmed by $\mathrm{Fe}$ addition from a few $L$ events. Thus, the possible range in $[\mathrm{Fe} / \mathrm{H}]_{P}$ has little effect on the determination of $n_{L}$ for MP stars. By using the above $P$-inventory and $L$ yield of $\mathrm{Fe}$, the value $n_{L}$ for a star can be obtained from equation (9) or from its equivalent in the $[\mathrm{Fe} / \mathrm{H}]$ notation,

$$
10^{[\mathrm{Fe} / \mathrm{H}]}=10^{[\mathrm{Fe} / \mathrm{H}] P}+n_{L} \times 10^{[\mathrm{Fe} / \mathrm{H}]_{L}} .
$$

In all our works, SN II $H$ and $L$ events are considered to occur stochastically with frequencies of $f_{H}$ and $f_{L}$ in some standard reference mass of hydrogen throughout the Galaxy over Galactic history (WBG96; WQ00; Qian \& Wasserburg 2000, 2001a; QW01). At any given time $t$ since the onset of SNe II, the probability $P\left(n_{H}, t\right)$ for a standard parcel of the ISM to have had a number $n_{H}$ of $H$ events is given by the Poisson distribution

$$
P\left(n_{H}, t\right)=\frac{\left(f_{H} t\right)^{n_{H}}}{n_{H} !} e^{-f_{H} t}=\frac{\left(\bar{n}_{H}\right)^{n_{H}}}{n_{H} !} e^{-\bar{n}_{H}},
$$

where $\bar{n}_{H}=f_{H} t$ is the average number of occurrences for $H$ events at time $t$. For $L$ events a similar expression applies:

$$
P\left(n_{L}, t\right)=\frac{\left(f_{L} t\right)^{n_{L}}}{n_{L} !} e^{-f_{L} t}=\frac{\left(\bar{n}_{L}\right)^{n_{L}}}{n_{L} !} e^{-\bar{n}_{L}} .
$$

The variances of the distributions in equations (11) and (12) are $\bar{n}_{H}$ and $\bar{n}_{L}$, respectively. The square root of the variance, $\bar{n}_{H}^{1 / 2}$ or $\bar{n}_{L}^{1 / 2}$, can be used as a measure of the variation in $n_{H}$ or $n_{L}$ at time $t: n_{H} \approx \bar{n}_{H} \pm \bar{n}_{H}^{1 / 2}$ or $n_{L} \approx \bar{n}_{L} \pm \bar{n}_{L}^{1 / 2}$. At $t=10^{9}$ yr, $n_{H} \approx 100 \pm 10$ corresponding to $\log \epsilon(\mathrm{Eu}) \approx-0.53$ to -0.44 , while $n_{L} \approx 10 \pm 3$ corresponding to $[\mathrm{Fe} / \mathrm{H}]$ $\approx-1.62$ to -1.36 . For shorter times, the dispersion in $n_{L}$ becomes much more prominent. For example, at $t=3 \times 10^{8} \quad \mathrm{yr}, \quad n_{H} \approx 30 \pm 5 \quad$ corresponding to $\log \epsilon(\mathrm{Eu}) \approx-1.08$ to -0.94 , while $n_{L} \approx 3 \pm 2$ corresponding to $[\mathrm{Fe} / \mathrm{H}] \approx-2.37$ to -1.76 . The abundances of heavy $r$-elements (e.g., Eu) are a better measure of time because of the higher frequency of $H$ events and hence much lower dispersion in $n_{H}$. Thus, it follows that there is a substantial dispersion in $[\mathrm{Fe} / \mathrm{H}]$ for a rather fixed abundance of any heavy $r$-element at any given time in the range $10^{8} \lesssim t \lesssim 2$ $\times 10^{9}$ yr. Conversely, a given $[\mathrm{Fe} / \mathrm{H}]$ value may correspond to a significant range of possible times. We consider that this effect causes the scatter in abundances of heavy $r$-elements at $-3 \lesssim[\mathrm{Fe} / \mathrm{H}]<-1.0$ with the dispersion rapidly increasing at lower $[\mathrm{Fe} / \mathrm{H}]$. The dispersion and mean trends for abundances of heavy and light $r$-elements in UMP and MP stars have been discussed in some detail by Qian (2001) based on the same approach.

As discussed in $\S 1.2$, the complex history of mixing and transport of elements in the ISM from which a star formed is represented by $n_{H}$ and $n_{L}$ for the star. It is shown above that these numbers can be calculated from the observed Eu and $\mathrm{Fe}$ abundances in the star by using the $H$ yield of $\mathrm{Eu}$ and the $L$ yield of Fe. Substituting the expressions for $n_{H}$ (eq. [8]) and $n_{L}$ (eq. [9] or eq. [10]) into the expression for the abundance of element $\mathrm{E}$ (eq. [3] or eq. [4]), we obtain

$$
\left(\frac{\mathrm{E}}{\mathrm{H}}\right)=\left(\frac{\mathrm{E}}{\mathrm{H}}\right)_{P}+\left(\frac{\mathrm{E}}{\mathrm{Eu}}\right)_{H}\left(\frac{\mathrm{Eu}}{\mathrm{H}}\right)+\left(\frac{\mathrm{E}}{\mathrm{Fe}}\right)_{L}\left[\left(\frac{\mathrm{Fe}}{\mathrm{H}}\right)-\left(\frac{\mathrm{Fe}}{\mathrm{H}}\right)_{P}\right] \text {. }
$$

As the yield of element $\mathrm{E}$ relative to $\mathrm{Eu}$ for $H$ events, $(\mathrm{E} / \mathrm{Eu})_{H}$, or to $\mathrm{Fe}$ for $L$ events, $(\mathrm{E} / \mathrm{Fe})_{L}$, is assumed to be fixed, the observed $\mathrm{Eu}$ or $\mathrm{Fe}$ abundance in the star contains all the prior history of mixing and transport for the part of element $\mathrm{E}$ coproduced with $\mathrm{Eu}$ in $H$ events or with $\mathrm{Fe}$ in $L$ events. Thus, the fundamental problem in our approach to understand abundances in UMP and MP stars is to establish the $P$-inventory composition and the yield templates of $H$ and $L$ events that underlie the observations. These results will be presented in the form of $\log \epsilon_{P}(\mathrm{E})$, $\log \epsilon_{H}(\mathrm{E})$, and $\log \epsilon_{L}(\mathrm{E})$ corresponding to $\log \epsilon_{P}(\mathrm{Fe}) \approx 4.51$ $([\mathrm{Fe} / \mathrm{H}] \approx-3), \log \epsilon_{H}(\mathrm{Eu})=-2.48$, and $\log \epsilon_{L}(\mathrm{Fe})=5.03$ $\left([\mathrm{Fe} / \mathrm{H}]_{L}=-2.48\right)$.

\subsection{Values of $n_{H}^{\odot}$ and $n_{L}^{\odot}$}

We assume here that the solar abundances are representative of an average parcel of the ISM at the time of SSF. We use the values $n_{H}^{\odot}=10^{3}$ and $n_{L}^{\odot}=10^{2}$ to account for the part of the solar inventory that was contributed by $\mathrm{SNe}$ II over a total uniform production time of $T_{\mathrm{UP}} \sim 10^{10} \mathrm{yr}$ prior to SSF. These values are directly related to the frequencies of $H$ and $L$ events that were derived from consideration of meteoritic data on the abundances of ${ }^{182} \mathrm{Hf}$ (with a lifetime $\left.\bar{\tau}_{182}=1.3 \times 10^{7} \mathrm{yr}\right)$ and ${ }^{129} \mathrm{I}\left(\bar{\tau}_{129}=2.3 \times 10^{7} \mathrm{yr}\right)$ in the ESS (WBG96). The meteoritic data require that replenishment of fresh ${ }^{182} \mathrm{Hf}$ in an average parcel of the ISM must occur on a timescale commensurate with its lifetime, which corresponds to a frequency of $f_{H} \sim\left(10^{7} \mathrm{yr}\right)^{-1}$ for $H$ events in a standard reference mass of hydrogen. As discussed in $\S 1.2$, this frequency can be explained by considering the Galactic SN II rate per unit mass of gas and the total amount of ISM typically swept up by an SN II remnant. Although there may be a range of possible values for $n_{H}^{\odot}=f_{H} T_{\mathrm{UP}}$, the observed abundances of heavy $r$ elements in UMP and MP stars appear to be well represented by the $H$ yields of these elements calculated by using $n_{H}^{\odot}=10^{3}$. For example, the lowest Eu abundances observed in such stars (e.g., McWilliam et al. 1995; Burris et al. 2000) are remarkably close to the Eu yield of a single $H$ event $\left[\log \epsilon_{H}(\mathrm{Eu})=-2.48\right]$ calculated from the solar $r$ inventory of Eu for $n_{H}^{\odot}=10^{3}$. In any case, a change in $n_{H}^{\odot}$ causes only a uniform shift in the $H$ yields of the relevant elements as the yield template is assumed here to be fixed. Such a shift will not affect the basic results presented here (see $\S \S 1.2$ and 1.3). 
The timescale for replenishment of fresh ${ }^{129} \mathrm{I}$ in an average parcel of the ISM required by the meteoritic data is much longer than its lifetime and corresponds to a frequency of $f_{L} \sim\left(10^{8} \mathrm{yr}\right)^{-1}$ for $L$ events. A decrease in the replenishment timescale (corresponding to an increase in $f_{L}$ ) would cause an exponential increase in the abundance of ${ }^{129} \mathrm{I}$ in the ESS. Many meteoritic measurements give a rather precise and very low value for this abundance $\left[\left({ }^{129} \mathrm{I} /{ }^{127} \mathrm{I}\right)_{\mathrm{ESS}}=10^{-4}\right.$; e.g., Brazzle et al. 1999], severely limiting any possible increase in $f_{L}$. Thus, we focus on the effect of decreasing $n_{L}^{\odot}=f_{L} T_{\mathrm{UP}}$ from $10^{2}$. For $n_{L}^{\odot}=10^{2}$ the $L$ yield of $\mathrm{Fe}$ is $[\mathrm{Fe} / \mathrm{H}]_{L}=-2.48$. A decrease in $n_{L}^{\odot}$ by a factor of 2 gives $[\mathrm{Fe} / \mathrm{H}]_{L}=-2.18$, which means that there would be a lack of stars between $[\mathrm{Fe} / \mathrm{H}] \approx-3$ and -2.18 . This is incompatible with the observations that show a continuous population at $[\mathrm{Fe} / \mathrm{H}] \gtrsim-2.5$. In any case, a change in $n_{L}^{\odot}$ again causes only a uniform shift in the $L$ yields of the relevant elements. Thus, changes in the values of $n_{H}^{\odot}$ and $n_{L}^{\odot}$ will not affect the basic conclusions of this work. But if the changes are large, they will violate the constraints indicated above. The standard values $n_{H}^{\odot}=10^{3}$ and $n_{L}^{\odot}=10^{2}$ will be used to derive the results in this work.

\subsection{The Solar r-Component}

In this study we are mainly concerned with the $r$-process. As discussed in $\S 1.1$, there must be at least two distinct types of $r$-process events. We associate both of these with SNe II, although they have not yet been proven to be the $r$-process sites. It was argued that the other candidate sites, such as neutron star mergers, would have difficulty explaining the observed $r$-abundances in UMP and MP stars if they were the principal source for $r$-nuclei (Qian 2000). Extensive and very sophisticated parametric studies of high neutron density environments have been carried out to gain insights into the conditions that are required for the $r$ process (Kratz et al. 1993; Hoffman, Woosley, \& Qian 1997; Meyer \& Brown 1997; Freiburghaus et al. 1999). These studies are usually directed toward approximating the overall solar $r$-abundance pattern by the $r$-process production in a prototypical environment. However, there is as yet no sufficient guidance from these studies to explain the distinct types of $r$-process events discussed in $\S 1.1$ or to make reliable a priori predictions for the templates of $r$ process yields ( $r$-yields) that can be used in Galactic chemical evolution models.

As also discussed in $\S 1.1$, the first generations of very massive stars formed from big bang debris produced a $P$ inventory of $\mathrm{Fe}$ and associated elements but very little heavy $r$-nuclei. The formation and evolution of these stars and their nucleosynthetic products have been a longstanding problem (e.g., Ezer \& Cameron 1971) and recently received renewed attention (e.g., Bromm, Coppi, \& Larson 1999; Heger et al. 2000; Fryer, Woosley, \& Heger 2001; Baraffe, Heger, \& Woosley 2001; Bromm et al. 2001). However, so far there is not sufficient information from theoretical models to predict the composition of the $P$ inventory assigned here to the first generations of stars.

Our approach here is to determine some of the $P$ inventory composition and the $r$-yields of SN II $H$ and $L$ events from the observed abundances in several selected stars based on the phenomenological model laid out in $\S \S 1.1-1.3$. These results will be used in the model to calculate the abundances for UMP and MP stars in general. The calculated abundances will then be compared with observations. One of the selected stars is the Sun, whose so-called cosmic abundance pattern (mostly derived from meteoritic studies) has served as the key to understanding nucleosynthesis (Burbidge et al. 1957; Cameron 1957). The part of the solar abundances required here is the $r$-process component ( $r$-component) that is obtained by subtracting the $s$-process component (s-component) from the total solar inventory.

One of the better understood processes of stellar nucleosynthesis is the s-process (see review by Busso, Gallino, \& Wasserburg 1999). The site of this process is well established observationally to be thermally pulsing AGB stars. Theoretical models for the $s$-process are based on the wellunderstood (but not completely) laws of stellar structure and evolution and the nuclear reactions in the stellar interior that produce the neutrons for slow capture by the preexisting seed nuclei. The nuclear physics involved both in neutron production and in s-processing has been the subject of extensive theoretical and experimental work. Many cross sections for important neutron capture reactions have been reliably measured. The classical analysis of the $s$-process relies on mainly the nuclear physics input and adopts a suitable distribution of neutron exposures that in principle can be calculated from stellar models. This approach is particularly successful for the so-called main $s$-component (see Käppeler, Beer, \& Wisshak 1989 for a review). The most recent $s$-process calculations of Arlandini et al. (1999) have emphasized the dependences of $s$-process yields on the initial mass and metallicity of AGB stars. Their calculations were embedded in very high quality stellar models with highly resolved structure of thermal pulses. They showed that the classical main $s$-component was very well reproduced by averaging the yields for models of 1.5 and $3 M_{\odot}$ AGB stars with $Z=0.5 Z_{\odot}(Z$ is the total mass fraction of all elements above $\mathrm{He}$, and $Z_{\odot}$ is the solar value).

However, there are still questions with regard to the onedimensional stellar models used in the new $s$-process calculations, such as the conditions obtained at critical boundary layers in AGB stars. One fundamental problem is the formation of the ${ }^{13} \mathrm{C}$ pocket that is the dominant source of neutrons [via ${ }^{13} \mathrm{C}(\alpha, n)^{16} \mathrm{O}$ ] in the new stellar models. By assuming that the mass of the ${ }^{13} \mathrm{C}$ pocket is constant for AGB stars with different metallicities, it was shown that the neutron-to-seed ratio for the $s$-process increases with decreasing metallicity, giving rise to preponderant production of heavier elements by low-metallicity AGB stars (Gallino et al. 1998). This result is very plausible. However, it appears to be in conflict with the recent observations of LP 625-44 (Aoki et al. 2000). This star has $[\mathrm{Fe} / \mathrm{H}]=-2.7$ and is enormously enriched in heavy elements from $\mathrm{Ba}$ and above. The high enrichments in LP 625-44 were attributed to surface contamination by mass transfer from an evolved AGB companion with the same metallicity $[\mathrm{Fe} / \mathrm{H}]=-2.7$ (Aoki et al. 2000) and do not reflect significant $s$-process addition to the ISM from which the star formed. The abundance pattern from $\mathrm{Ba}$ and above in this star matches that of the main $s$-component calculated by Arlandini et al. (1999) from models of AGB stars but with $Z=0.5 Z_{\odot}$. As pointed out by Aoki et al. (2000), this abundance pattern is not in accord with models of low-metallicity AGB stars that have very high neutron-to-seed ratios: the observed $\mathrm{Pb} / \mathrm{Ba}$ ratio is lower by 2 orders of magnitude than the value predicted by Busso et al. (1999) from stellar models with 
$[\mathrm{Fe} / \mathrm{H}]=-2.7$. On the other hand, the observed abundances of $\mathrm{Sr}, \mathrm{Y}$, and $\mathrm{Zr}$ relative to $\mathrm{Ba}$ are a few orders of magnitude lower than what would be expected for stellar models with $Z=0.5 Z_{\odot}$. The s-process in low-metallicity AGB stars thus remains open to investigation, particularly with regard to the magnitude of the ${ }^{13} \mathrm{C}$ pocket. We are fully aware that there have been remarkable advances in models of AGB evolution and s-process nucleosynthesis over the past decade (see Busso et al. 1999 and references therein). Our purpose in the above discussion is not to decry the status of that field but to call attention to some uncertainties critical to our study.

The solar $r$-abundance of element $\mathrm{E}$ is calculated as

$$
\left(\frac{\mathrm{E}}{\mathrm{H}}\right)_{\odot, r}=\left(\frac{\mathrm{E}}{\mathrm{H}}\right)_{\odot}-\left(\frac{\mathrm{E}}{\mathrm{H}}\right)_{\odot, s} \equiv\left(1-\beta_{\odot, s}\right)\left(\frac{\mathrm{E}}{\mathrm{H}}\right)_{\odot} .
$$

In all cases in which $\beta_{\odot, s}(\mathrm{E})$ is small, the results for $(\mathrm{E} / \mathrm{H})_{\odot, r}$ are sound and provide the basis for comparison of the observed $r$-abundance patterns in UMP and MP stars with the solar $r$-component. In this work we are concerned with $\mathrm{Sr}, \mathrm{Y}, \mathrm{Zr}$, and $\mathrm{Ba}$ for which $\beta_{\odot, s} \sim 1$ with substantial uncertainties (Käppeler et al. 1989; Arlandini et al. 1999). For example, the value for $(\mathrm{Sr} / \mathrm{H})_{\odot, r}$ assigned by Käppeler et al. (1989) has an uncertainty of $111 \%$ and is larger by factors of $\approx 2-3$ than the values given by Arlandini et al. (1999). For $\mathrm{Y}$, the calculated s-process contribution exceeds its total solar inventory in the classical analysis by Arlandini et al. (1999), and their stellar model calculations give a value for $(\mathrm{Y} / \mathrm{H})_{\odot, r}$ that is smaller by a factor of 3.5 than the value assigned by Käppeler et al. (1989). Further, the division of the solar inventory into $s$-components and $r$-components is not well defined for $\mathrm{Sr}, \mathrm{Y}$, and $\mathrm{Zr}$ as these elements can also be produced by the $\alpha$-process that does not involve neutron capture but may occur in close connection with the $r$ process in SNe II (Woosley \& Hoffman 1992). In the case of Ba, Käppeler et al. (1989) gave only an upper limit for $\left({ }^{138} \mathrm{Ba} / \mathrm{H}\right)_{\odot, r}$, and Arlandini et al. (1999) have already found shifts in the main $s$-component of $\mathrm{Ba}$ that result in a range of $\left[1-\beta_{\odot, s}(\mathrm{Ba})\right]$ from 0.08 in the classical analysis to 0.19 in the stellar model calculations. This clearly illustrates that care must be taken in using the solar $r$-abundances when the corresponding $\beta_{\odot, s}$ values are close to unity.

The $\beta_{\odot, s}$ values for the elements $\mathrm{Y}$ and above that are used here correspond to the main $s$-component calculated by Arlandini et al. (1999) from stellar models. These results represent a significant step forward from the phenomenological approach of the classical analysis, but they do not represent a full calculation of the integrated s-process production by AGB stars over Galactic history. In addition to the main s-component, the classical analysis also introduces a so-called weak s-component that is important for $\mathrm{Sr}$ and below (e.g., Käppeler et al. 1989). The $\beta_{\odot, s}$ value for $\mathrm{Sr}$ used here is also taken from the stellar model calculations of Arlandini et al. (1999) but includes the contribution from the weak s-component considered by these authors. The $\beta_{\odot, s}$ values cited above and adopted for our initial calculations will be shown to result in a mismatch between the calculated $\mathrm{Sr}, \mathrm{Y}, \mathrm{Zr}$, and $\mathrm{Ba}$ abundances and the observational data on MP stars. It is widely recognized in the field that the abundances of $\mathrm{Sr}, \mathrm{Y}$, and $\mathrm{Zr}$ are problematic, particularly at low metallicities, relative to any current nucleosynthetic models (M. Busso \& R. Gallino 2001, private communication). While we also explore other explanations for the mismatch between our model results and data (see $\S 4$ ), we feel that it is reasonable to consider the uncertainties in the solar s-component for $\mathrm{Sr}, \mathrm{Y}, \mathrm{Zr}$, and $\mathrm{Ba}$. It is possible that the same uncertainties may also affect other elements whose $\beta_{\odot, s}$ values are not small.

\section{THE $P$-INVENTORY AND $H$ AND $L$ YIELDS}

The $P$-inventory and $H$ and $L$ yields are the key input to our model. These are calculated in this section.

\subsection{The P- and H-Components}

As addition of $\mathrm{Fe}$ from a single $L$ event to the $P$ inventory would give $[\mathrm{Fe} / \mathrm{H}] \approx-2.4$ (see eq. $[10]$ ), UMP stars with $[\mathrm{Fe} / \mathrm{H}] \approx-3$ could not have received any contributions from $L$ events (i.e., $n_{L}=0$ ). Thus, the abundances in these stars reflect only the addition of $H$ contributions to the $P$-inventory, providing a unique record for the $P$ - and $H$-components. Equation (4) can be simplified for UMP stars as

$$
10^{\log \epsilon(\mathrm{E})}=10^{\log \epsilon P(\mathrm{E})}+n_{H} \times 10^{\log \epsilon_{H}(\mathrm{E})} .
$$

The value $n_{H}$ for a star can be calculated from the observed $\mathrm{Eu}$ abundance in the star by using equation (8). For example, Eu data on the UMP stars HD 122563, HD 115444, and CS 22892-052 (Westin et al. 2000; Sneden et al. 2000) correspond to $n_{H} \approx 1,7$, and 36 (see Table 2). The abundances of element $E$ other than Eu in two UMP stars with different $n_{H}$ provide a pair of equations in the form of equation (15) with two unknown parameters $\log \epsilon_{P}(\mathrm{E})$ and $\log \epsilon_{H}(\mathrm{E})$. Thus, the data on two such UMP stars (singly and doubly primed quantities) can be used to determine simultaneously the $P$-inventory and $H$ yield of element $\mathrm{E}$ :

$$
\begin{aligned}
10^{\log \epsilon(\mathrm{E})} & =\frac{n_{H}^{\prime} \times 10^{\log \epsilon^{\prime \prime}(\mathrm{E})}-n_{H}^{\prime \prime} \times 10^{\log \epsilon^{\prime}(\mathrm{E})}}{n_{H}^{\prime}-n_{H}^{\prime \prime}}, \\
10^{\log \epsilon_{H}(\mathrm{E})} & =\frac{10^{\log \epsilon^{\prime}(\mathrm{E})}-10^{\log \epsilon^{\prime \prime}(\mathrm{E})}}{n_{H}^{\prime}-n_{H}^{\prime \prime}} .
\end{aligned}
$$

The results for $\mathrm{Sr}, \mathrm{Y}$, and $\mathrm{Zr}$ obtained by using the data (see Table 2) on HD $115444([\mathrm{Fe} / \mathrm{H}]=-2.99)$ and CS 22892 $052([\mathrm{Fe} / \mathrm{H}]=-3.1)$ are given in Table 3 .

The $P$-component of $\mathrm{Sr}, \mathrm{Y}$, and $\mathrm{Zr}$ is associated with the prompt production of $\mathrm{Fe}$. Thus, any difference in $[\mathrm{Fe} / \mathrm{H}]$ among UMP stars should also be reflected by the $P$ component of these elements (see below). This indicates

TABLE 2

Data on Three UMP Stars and Calculated Numbers of $H$ and $L$ Events

\begin{tabular}{ccccccccc}
\hline \hline Star & {$[\mathrm{Fe} / \mathrm{H}]$} & $n_{L}$ & $\log \epsilon(\mathrm{Eu})$ & $n_{H}$ & $\log \epsilon(\mathrm{Sr})$ & $\log \epsilon(\mathrm{Y})$ & $\log \epsilon(\mathrm{Zr})$ & Reference \\
\hline HD 122563 ........ & -2.74 & 0 & -2.59 & 1 & 0.33 & -0.75 & 0.04 & 1 \\
HD 115444 ....... & -2.99 & 0 & -1.63 & 7 & 0.23 & -0.82 & -0.02 & 1 \\
CS 22892-052...... & -3.1 & 0 & -0.93 & 36 & 0.50 & -0.39 & 0.26 & 2 \\
\hline
\end{tabular}

REFERENCES.- (1) Westin et al. 2000; (2) Sneden et al. 2000. 
TABLE 3

PARAMETERS For THE THREe-COMPONENT MODEL

\begin{tabular}{|c|c|c|c|c|c|c|c|c|c|}
\hline $\begin{array}{l}Z^{\mathrm{a}} \\
(1)\end{array}$ & $\begin{array}{l}\mathrm{E} \\
(2)\end{array}$ & $\begin{array}{c}\log \epsilon_{P}(E) \\
\text { (3) }\end{array}$ & $\begin{array}{c}\log \epsilon_{H}(\mathrm{E}) \\
\text { (4) }\end{array}$ & $\begin{array}{c}\log \epsilon_{L}(\mathrm{E}) \\
(5)\end{array}$ & $\begin{array}{c}\log \epsilon_{\odot, r}(\mathrm{E}) \\
(6)\end{array}$ & $\begin{array}{c}\beta_{\odot, s}(\mathrm{E}) \\
(7)\end{array}$ & $\begin{array}{c}\log \epsilon_{L}^{\text {corr }}(E) \\
(8)\end{array}$ & $\begin{array}{c}\log \epsilon_{\odot, r}^{\text {corr }}(\mathrm{E}) \\
(9)\end{array}$ & $\begin{array}{c}\beta_{\odot, s}^{\text {corr }}(E) \\
(10)\end{array}$ \\
\hline $26 \ldots \ldots$ & $\mathrm{Fe}^{\mathrm{b}}$ & 4.51 & $-\infty$ & 5.03 & $\ldots$ & $\ldots$ & $\ldots$ & $\ldots$ & $\ldots$ \\
\hline $38 \ldots \ldots$ & $\mathrm{Sr}$ & 0.13 & -1.30 & $-\infty^{c}$ & 1.64 & $0.95^{\mathrm{d}}$ & 0.35 & 2.44 & 0.69 \\
\hline $39 \ldots \ldots$ & $\mathrm{Y}$ & -1.05 & -2.05 & -1.36 & 1.12 & 0.92 & -0.42 & 1.67 & 0.72 \\
\hline $40 \ldots \ldots$ & $\mathrm{Zr}$ & -0.13 & -1.53 & -0.40 & 1.85 & 0.83 & 0.26 & 2.32 & 0.49 \\
\hline $41 \ldots \ldots$ & $\mathrm{Nb}$ & $-\infty$ & -2.56 & -2.06 & 0.56 & 0.85 & $\ldots$ & $\ldots$ & $\ldots$ \\
\hline $44 \ldots \ldots$ & $\mathrm{Ru}$ & $-\infty$ & -1.56 & -0.92 & 1.60 & 0.32 & $\ldots$ & $\ldots$ & $\ldots$ \\
\hline $45 \ldots \ldots$ & $\mathrm{Rh}$ & $-\infty$ & -2.36 & -1.20 & 1.03 & 0.14 & $\ldots$ & $\ldots$ & $\ldots$ \\
\hline $46 \ldots \ldots$ & $\mathrm{Pd}$ & $-\infty$ & -1.82 & -0.95 & 1.42 & 0.46 & $\ldots$ & $\ldots$ & $\ldots$ \\
\hline $47 \ldots \ldots$ & $\mathrm{Ag}$ & $-\infty$ & -2.36 & -1.02 & 1.14 & 0.20 & $\ldots$ & $\ldots$ & $\ldots$ \\
\hline $48 \ldots \ldots$ & $\mathrm{Cd}$ & $-\infty$ & -1.91 & -0.85 & 1.42 & 0.52 & $\ldots$ & $\ldots$ & $\ldots$ \\
\hline $56 \ldots \ldots$ & $\mathrm{Ba}$ & $-\infty$ & $-1.52^{\mathrm{e}}$ & $-\infty$ & 1.48 & 0.81 & -0.47 & 1.79 & 0.62 \\
\hline $57 \ldots \ldots$ & $\mathrm{La}$ & $-\infty$ & -2.22 & $-\infty$ & 0.78 & 0.62 & $\ldots$ & $\ldots$ & $\ldots$ \\
\hline $58 \ldots \ldots$ & $\mathrm{Ce}$ & $-\infty$ & -2.02 & $-\infty$ & 0.98 & 0.77 & $\ldots$ & $\ldots$ & $\ldots$ \\
\hline $59 \ldots \ldots$ & Pr & $-\infty$ & -2.51 & $-\infty$ & 0.49 & 0.49 & $\ldots$ & $\ldots$ & $\ldots$ \\
\hline $60 \ldots \ldots$ & $\mathrm{Nd}$ & $-\infty$ & -1.90 & $-\infty$ & 1.10 & 0.56 & $\ldots$ & $\ldots$ & $\ldots$ \\
\hline $62 \ldots \ldots$ & $\mathrm{Sm}$ & $-\infty$ & -2.20 & $-\infty$ & 0.80 & 0.29 & $\ldots$ & $\ldots$ & $\ldots$ \\
\hline $63 \ldots \ldots$ & $\mathrm{Eu}$ & $-\infty$ & -2.48 & $-\infty$ & 0.52 & 0.06 & $\ldots$ & $\ldots$ & $\ldots$ \\
\hline $64 \ldots \ldots$ & $\mathrm{Gd}$ & $-\infty$ & -2.00 & $-\infty$ & 1.00 & 0.15 & $\ldots$ & $\ldots$ & $\ldots$ \\
\hline $65 \ldots \ldots$ & $\mathrm{Tb}$ & $-\infty$ & -2.70 & $-\infty$ & 0.30 & 0.07 & $\ldots$ & $\ldots$ & $\ldots$ \\
\hline $66 \ldots \ldots$ & Dy & $-\infty$ & -1.92 & $-\infty$ & 1.08 & 0.15 & $\ldots$ & $\ldots$ & $\ldots$ \\
\hline $67 \ldots \ldots$ & Ho & $-\infty$ & -2.53 & $-\infty$ & 0.47 & 0.08 & $\ldots$ & $\ldots$ & $\ldots$ \\
\hline $68 \ldots \ldots$ & $\mathrm{Er}$ & $-\infty$ & -2.13 & $-\infty$ & 0.87 & 0.17 & $\ldots$ & $\ldots$ & $\ldots$ \\
\hline $69 \ldots \ldots$ & $\mathrm{Tm}$ & $-\infty$ & -2.93 & $-\infty$ & 0.07 & 0.13 & $\ldots$ & $\ldots$ & $\ldots$ \\
\hline $70 \ldots \ldots$ & $\mathrm{Yb}$ & $-\infty$ & -2.22 & $-\infty$ & 0.78 & 0.33 & $\ldots$ & $\ldots$ & $\ldots$ \\
\hline $71 \ldots \ldots$ & $\mathrm{Lu}$ & $-\infty$ & -2.98 & $-\infty$ & 0.02 & 0.20 & $\ldots$ & $\ldots$ & $\ldots$ \\
\hline $72 \ldots \ldots$ & $\mathrm{Hf}$ & $-\infty$ & -2.61 & $-\infty$ & 0.39 & 0.56 & $\begin{array}{l}\cdots \\
\ldots\end{array}$ & $\begin{array}{l}\cdots \\
\ldots\end{array}$ & $\begin{array}{l}\cdots \\
\ldots\end{array}$ \\
\hline $73 \ldots \ldots$ & $\mathrm{Ta}$ & $-\infty$ & -3.36 & $-\infty$ & -0.36 & 0.41 & $\ldots$ & $\ldots$ & $\ldots$ \\
\hline $74 \ldots \ldots$ & $\mathrm{W}$ & $-\infty$ & -2.68 & $-\infty$ & 0.32 & 0.56 & $\ldots$ & $\ldots$ & $\ldots$ \\
\hline $75 \ldots \ldots$ & $\operatorname{Re}$ & $-\infty$ & -2.75 & $-\infty$ & 0.25 & 0.09 & $\ldots$ & $\ldots$ & $\ldots$ \\
\hline $76 \ldots \ldots$ & Os & $-\infty$ & -1.66 & $-\infty$ & 1.34 & 0.09 & $\ldots$ & $\ldots$ & $\ldots$ \\
\hline $77 \ldots \ldots$ & $\mathrm{Ir}$ & $-\infty$ & -1.63 & $-\infty$ & 1.37 & 0.01 & $\ldots$ & $\ldots$ & $\ldots$ \\
\hline $78 \ldots \ldots$ & $\mathrm{Pt}$ & $-\infty$ & -1.34 & $-\infty$ & 1.66 & 0.05 & $\ldots$ & $\ldots$ & $\ldots$ \\
\hline $79 \ldots \ldots$ & $\mathrm{Au}$ & $-\infty$ & -2.20 & $-\infty$ & 0.80 & 0.06 & $\ldots$ & $\ldots$ & $\ldots$ \\
\hline $90 \ldots \ldots$ & ${ }^{232} \mathrm{Th}^{\mathrm{f}}$ & $-\infty$ & -2.72 & $-\infty$ & 0.18 & 0 & $\cdots$ & $\cdots$ & $\cdots$ \\
\hline $92 \ldots \ldots$ & ${ }^{238} \mathrm{U}^{\mathrm{f}}$ & $-\infty$ & -2.89 & $-\infty$ & -0.19 & 0 & $\ldots$ & $\ldots$ & $\ldots$ \\
\hline
\end{tabular}

Note. - The $P$-inventory in col. (3) and $H$ yields in col. (4) are calculated from the data on HD 115444 and CS 22892-052 in Table 1 for $\mathrm{Sr}, \mathrm{Y}$, and $\mathrm{Zr}$. The $P$-inventory for elements above $\mathrm{Zr}$ is negligible and set to $-\infty$. The other $H$ yields are calculated from the data on CS 22892-052 for Nb to Cd and from the standard solar $r$-abundances (Arlandini et al. 1999) in col. (6) (corresponding to the solar s-process fractions in col. [7]) for Ba to $\mathrm{Au}$. The $L$ yields in col. (5) are calculated from cols. (3), (4), and (6) for $\mathrm{Sr}$ to $\mathrm{Cd}$ and set to $-\infty$ for $\mathrm{Ba}$ and above. Cols. (8)-(10) give the corrected values proposed here for $\mathrm{Sr}, \mathrm{Y}, \mathrm{Zr}$, and $\mathrm{Ba}$.

${ }^{a}$ Atomic number.

${ }^{b}$ Use $\log \epsilon_{\odot}(\mathrm{Fe})=7.51$ to obtain $[\mathrm{Fe} / \mathrm{H}] \equiv \log \epsilon(\mathrm{Fe})-\log \epsilon_{\odot}(\mathrm{Fe})$. The $P$-inventory and $H$ and $L$ yields of $\mathrm{Fe}$ are inferred from the data on UMP stars and the part of the solar Fe inventory contributed by SNe II.

${ }^{c}$ The standard solar $r$-inventory of $\mathrm{Sr}$ is saturated by the contributions from $n_{H}^{\odot}=10^{3} \mathrm{H}$ events and thus does not allow any $L$ contributions to $\mathrm{Sr}$.

${ }^{\mathrm{d}}$ This $\beta_{\odot, s}(\mathrm{Sr})$ value is based on the solar $r$-process fraction of $\left[1-\beta_{\odot, s}(\mathrm{Sr})\right]=0.05$ given by Arlandini et al. 1999 .

e This $H$ yield of $\mathrm{Ba}$ is calculated by attributing the standard solar $r$-inventory of $\mathrm{Ba}$ to $n_{H}^{\odot}=10^{3} H$ events and is essentially identical to the value of $\log \epsilon_{H}(\mathrm{Ba}) \approx-1.57$ obtained from the data on CS 22892-052. The latter value is used in the calculations.

${ }^{\mathrm{f}}$ The $H$ yields of ${ }^{232} \mathrm{Th}$ and ${ }^{238} \mathrm{U}$ are taken from the calculations of QW01 based on the inventory of these two nuclei in the ESS (given here as the $\log \epsilon_{\odot, r}$ values).

that the $P$-inventory of $\mathrm{Sr}, \mathrm{Y}$, and $\mathrm{Zr}$ for HD 122563 with $[\mathrm{Fe} / \mathrm{H}]=-2.74$ should be $\approx 0.3$ dex higher than the $\log \epsilon_{P}$ values for these elements calculated above by using the data on HD 115444 and CS 22892-052, which have lower [Fe/H] values (by $\approx 0.3 \mathrm{dex}$ ). The observed Eu abundance in HD 122563 gives $n_{H} \approx 1$ for this star (see Table 2). The $H$ yields of $\mathrm{Sr}, \mathrm{Y}$, and $\mathrm{Zr}$ calculated above are low, which suggests that the abundances of these elements in HD 122563 should essentially reflect their $P$-inventory for this star. The observed abundances of Sr, Y, and $\mathrm{Zr}$ in HD 122563 (see Table 2 ) are indeed $\approx 0.2-0.3$ dex higher than the $\log \epsilon_{P}$ values in Table 3.
The level of metallicity necessary for the formation of the first generation of normal stars is not known a priori. We have inferred from the observational data that normal astration first occurred at $[\mathrm{Fe} / \mathrm{H}] \approx-3$ based on the phenomenological model. Recently, Bromm et al. (2001) have shown that a gas cloud with a baryon mass of $10^{5} M_{\odot}$ and $Z=10^{-4} Z_{\odot}$ fails to undergo continued collapse and fragmentation whereas for $Z=10^{-3} Z_{\odot}$ the cloud can collapse and undergo vigorous fragmentation, leading to a large relative population of low-mass clumps compared with the case of $Z=0$ (big bang debris). The phenomenological and dynamical views thus appear to be in accord for 
the moment. The difference in $[\mathrm{Fe} / \mathrm{H}]$ among UMP stars referred to in our above discussion may have resulted from a range of values for the $P$-inventory of $\mathrm{Fe}$, possibly as wide as $-4<[\mathrm{Fe} / \mathrm{H}]_{P}<-2.7$, over which normal astration may first have occurred. We have chosen a nominal value of $[\mathrm{Fe} / \mathrm{H}]_{P} \approx-3$ in the above calculation of the $P$ component. As we assign no Eu or any other heavy $r$ element to the $P$-inventory, the possible range of $[\mathrm{Fe} / \mathrm{H}]_{P}$ does not affect the calculation of $n_{H}$ nor that of $\log \epsilon(\mathrm{E})$ for heavy $r$-elements produced by $H$ events. However, elements such as $\mathrm{Sr}, \mathrm{Y}$, and $\mathrm{Zr}$ associated with the $P$-inventory are directly related to the prompt $\mathrm{Fe}$ production. It follows that $\log \epsilon_{P}(\mathrm{E})$ for these elements will be shifted with $\log \epsilon_{P}(\mathrm{Fe})$ depending on the precise value of $[\mathrm{Fe} / \mathrm{H}]$ at which normal astration began. In comparing the $P$-components of element $\mathrm{E}$ for UMP stars, it is therefore necessary to correct to a standard value of $[\mathrm{Fe} / \mathrm{H}]$ in order to obtain the $P$ inventory of element $\mathrm{E}$ relative to $\mathrm{Fe}$. The $\log \epsilon_{P}(\mathrm{E})$ values in Table 3 should be associated with $[\mathrm{Fe} / \mathrm{H}]_{P} \approx-3$. If a UMP star has $[\mathrm{Fe} / \mathrm{H}]=-3+\delta$, then its $P$-component has to be shifted by $\delta$ from the tabulated $\log \epsilon_{P}(\mathrm{E})$ values as was done above for HD 122563.

We have assigned the heavy $r$-elements above $\mathrm{Ba}$ (possibly including $\mathrm{Ba}$ ) exclusively to $H$ events with a yield template that is identical to the corresponding part of the solar $r$-abundance pattern (see $\S 3.1$ ). For these elements, including $\mathrm{Eu}$ whose observed abundance is used to calculate $n_{H}$ for a star, $\log \epsilon_{P}(\mathrm{E})=-\infty$ and $\log \epsilon_{H}(\mathrm{E})=\log \epsilon_{\odot, r}(\mathrm{E})$ $-\log n_{H}^{\odot}$, where $n_{H}^{\odot}=10^{3}$ is the number of $H$ events that contributed to the solar inventory. The $H$ yields of $\mathrm{Ba}$ to $\mathrm{Au}$ calculated this way have been given by QW01 and are repeated in Table 3. The $H$ yields of heavy $r$-elements other than Eu may also be directly calculated from the observed abundances of these elements in any UMP star without using their solar $r$-inventory. For example, CS 22892-052 with $n_{H}^{\mathrm{CS}} \approx 36$ (see Table 2) has $\log \epsilon_{\mathrm{CS}}(\mathrm{Ba})=-0.01$ (Sneden et al. 2000). This gives $\log \epsilon_{H}(\mathrm{Ba})=\log \epsilon_{\mathrm{CS}}(\mathrm{Ba})-\log n_{H}^{\mathrm{CS}} \approx$ -1.57 in excellent agreement with the value $\log \epsilon_{H}(\mathrm{Ba})=$ $\log \epsilon_{\odot, r}(\mathrm{Ba})-\log n_{H}^{\odot}=-1.52$ obtained above for $\log \epsilon_{\odot, r}(\mathrm{Ba})=1.48$ (Arlandini et al. 1999). The long-lived chronometers ${ }^{232} \mathrm{Th}$ and ${ }^{238} \mathrm{U}$ are produced along with the stable elements above $\mathrm{Ba}$ (possibly including $\mathrm{Ba}$ ) in $H$

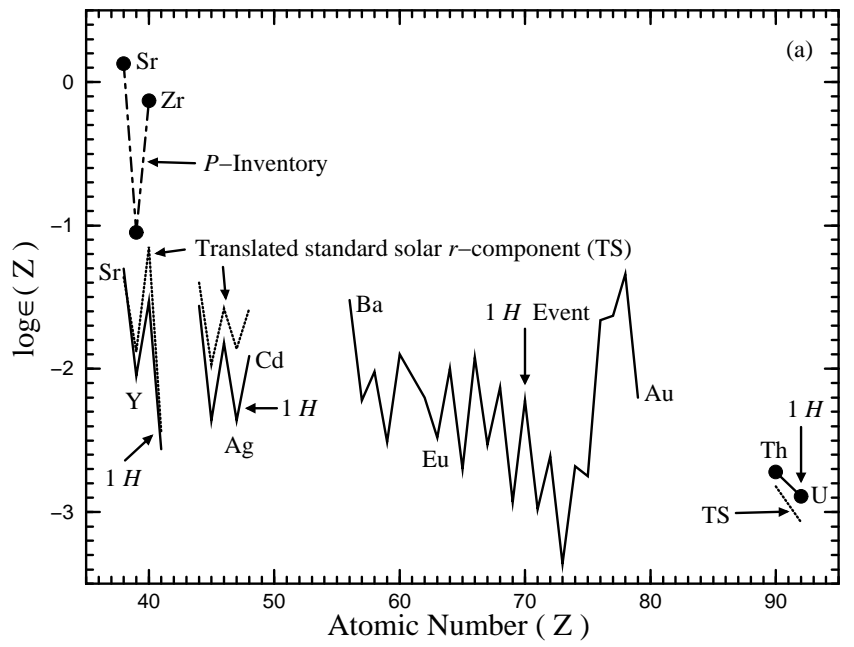

events. The $H$ yields of these two nuclei have been calculated by QW01 based on their solar $r$-abundances and are given in Table 3. Of the stable elements above $\mathrm{Au}$, only $\mathrm{Pb}$ has been observed in UMP and MP stars with large uncertainties (e.g., Sneden et al. 2000). The $H$ yields of these elements are not given here.

Besides $\mathrm{Sr}, \mathrm{Y}$, and $\mathrm{Zr}$, the light $r$-elements $\mathrm{Nb}, \mathrm{Ru}, \mathrm{Rh}$, $\mathrm{Pd}, \mathrm{Ag}$, and Cd have been observed in the UMP star CS 22892-052, but with significantly lower abundances relative to the solar $r$-abundance pattern that is translated to pass through the Eu data (Sneden et al. 2000). This suggests that some light $r$-elements may be produced along with the heavy $r$-elements in $H$ events, but with relative yields of light to heavy $r$-elements significantly smaller than the corresponding solar $r$-abundance ratios. If we consider that the $P$-inventory of $\mathrm{Nb}, \mathrm{Ru}, \mathrm{Rh}, \mathrm{Pd}, \mathrm{Ag}$, and $\mathrm{Cd}$ is overwhelmed by contributions from the $n_{H}^{\mathrm{CS}} \approx 36 \mathrm{H}$ events assigned to CS 22892-052 (see Table 2), then the $H$ yields of these elements can be estimated from their observed abundances, $\log \epsilon_{\mathrm{CS}}(\mathrm{E}), \quad$ as $\quad \log \epsilon_{H}(\mathrm{E}) \approx \log \epsilon_{\mathrm{CS}}(\mathrm{E})-\log n_{H}^{\mathrm{CS}}$. Similar results were obtained by QW01, who used the observed abundance of Os instead of Eu to calculate $n_{H}^{\mathrm{CS}}$. The $P$ inventory and $H$ yields derived above are summarized in Table 3 and shown in Figure 2.

\subsection{The L-Component}

As discussed in $\S 2.1$, the $P$-inventory and $H$ yields are calculated from data on two UMP stars with different $n_{H}$ values. In this calculation, only the solar $r$-inventory of $\mathrm{Eu}$ is needed to obtain the $H$ yield of Eu. The yields for the other elements can be directly obtained from observations of UMP stars although the yields of the other heavy $r$ elements can also be calculated by using their solar $r$ inventory. In contrast, the $L$ yields will be calculated by using the solar $r$-inventory as an essential input. This inventory was contributed by $n_{H}^{\odot}=10^{3} H$ and $n_{L}^{\odot}=10^{2} L$ events in addition to the $P$-inventory:

$$
\left(\frac{\mathrm{E}}{\mathrm{H}}\right)_{\odot, r}=\left(\frac{\mathrm{E}}{\mathrm{H}}\right)_{P}+n_{H}^{\odot}\left(\frac{\mathrm{E}}{\mathrm{H}}\right)_{H}+n_{L}^{\odot}\left(\frac{\mathrm{E}}{\mathrm{H}}\right)_{L} .
$$

The solar $r$-abundance $\log \epsilon_{\odot, r}(\mathrm{E})$ given in Table 3 (Arlandini et al. 1999; see $\S 1.5$ ) is used in equation (17) to

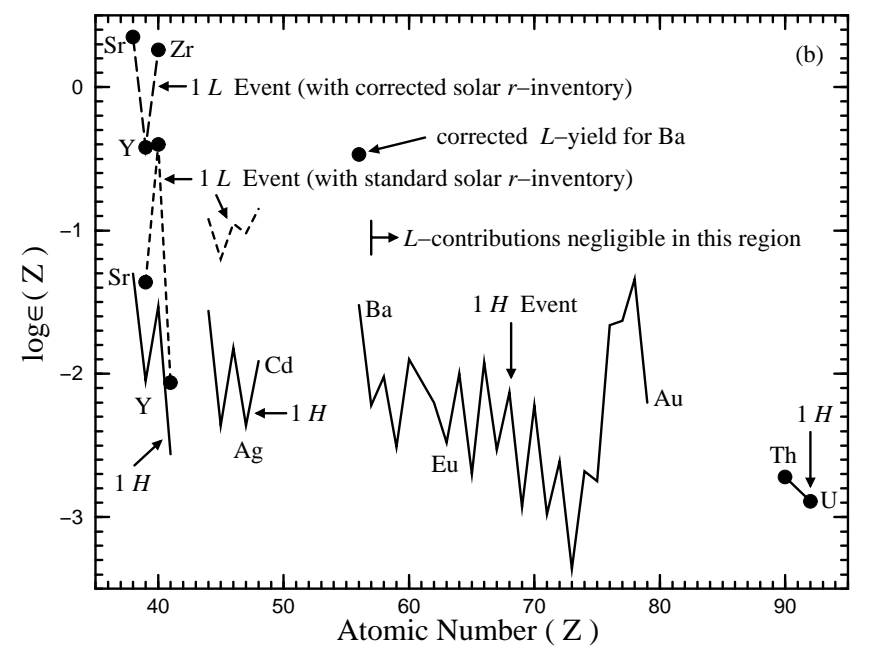

FIG. 2.- $P$-inventory and $H$ and $L$ yields (see Table 3). (a) $P$-inventory (dot-dashed curve), the $H$ yields (solid curve), and the standard solar $r$-component (dotted curve) that is translated to coincide with the $H$ yields of $\mathrm{Ba}$ to $\mathrm{Au}$. (b) $L$ yields corresponding to the standard (short-dashed curve) and corrected (longdashed curve) solar $r$-inventory and the $H$ yields (solid curve). The $L$ yields of $\mathrm{Sr}$ and Ba only exist for the corrected solar $r$-inventory of these two elements. The $L$ contributions to elements above $\mathrm{Ba}$ are negligible. 
calculate the $L$ yield of element $\mathrm{E}$ from the $\log \epsilon_{P}(\mathrm{E})$ and $\log \epsilon_{H}(E)$ values in Table 3. The resulting $L$ yields from this calculation are also given in Table 3 and shown in Figure 2. Note that $\log \epsilon_{L}(E)=-\infty$ for the heavy $r$-elements above $\mathrm{Ba}$ (possibly including $\mathrm{Ba}$ ) as they have been exclusively assigned to $H$ events (see $\S 2.1$ ).

\section{COMPARISON OF MODEL RESULTS WITH OBSERVATIONAL DATA}

The $\log \epsilon_{P}(\mathrm{E}), \log \epsilon_{H}(\mathrm{E})$, and $\log \epsilon_{L}(\mathrm{E})$ values in Table 3 permit us to calculate the abundance of element $E$ in any UMP or MP star from the model described in $\S 1$ by using only the observed $\mathrm{Eu}$ and $\mathrm{Fe}$ abundances to obtain $n_{H}$ and $n_{L}$ for the star (see eqs. [4], [8], and [10]). The calculated abundances are compared with the observational data in this section.

\subsection{Abundances of $\mathrm{Ba}$ and Above in UMP and MP Stars}

The comparison of model results with observational data is the simplest for the heavy $r$-elements above $\mathrm{Ba}$ (possibly including $\mathrm{Ba}$ ) as they have been exclusively assigned to $H$
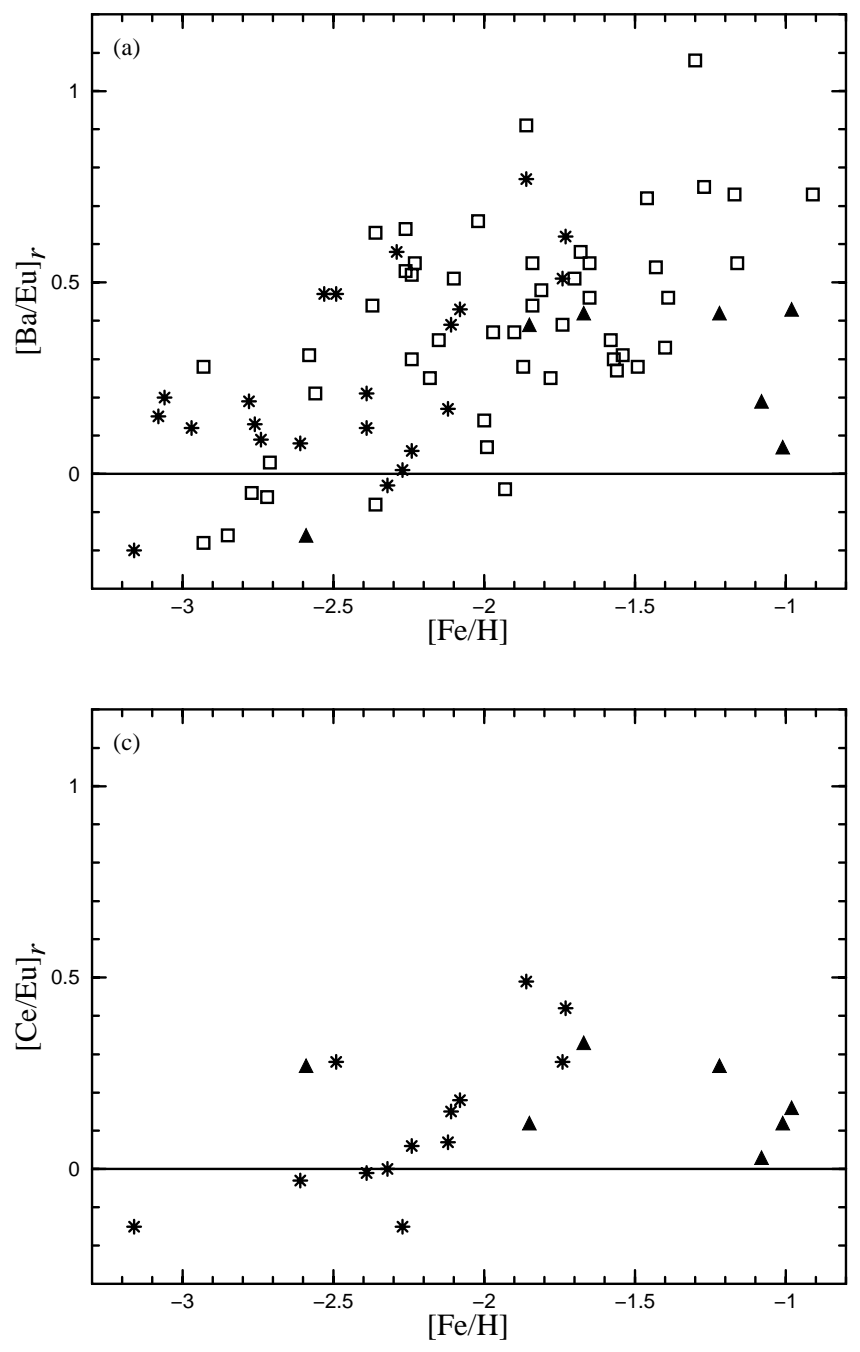

events with a yield template identical to the corresponding part of the solar $r$-abundance pattern (see $\S 2.1$ ). As a test of this hypothesis, we consider the abundance ratios of other heavy $r$-elements (E) to Eu. These ratios should remain at the solar $r$-process values independent of $[\mathrm{Fe} / \mathrm{H}]$ (i.e., the occurrence of $L$ events) if the heavy $r$-elements come only from $H$ events. Based on the data of Burris et al. (2000; open squares), Johnson \& Bolte (2001; asterisks), and Gratton \& Sneden (1994; filled triangles), the difference between the observed $\mathrm{E} / \mathrm{Eu}$ and the solar $r$-process value of Arlandini et al. (1999), $[\mathrm{E} / \mathrm{Eu}]_{r} \equiv \log (\mathrm{E} / \mathrm{Eu})-\log (\mathrm{E} / \mathrm{Eu})_{\odot, r}$, is shown as a function of $[\mathrm{Fe} / \mathrm{H}]$ for $\mathrm{Ba}, \mathrm{La}, \mathrm{Ce}, \mathrm{Pr}, \mathrm{Nd}, \mathrm{Sm}, \mathrm{Gd}$, and Dy in Figure 3. The data sets of Burris et al. (2000) and Johnson \& Bolte (2001) have 17 stars in common. Figure 3 shows that (1) $\mathrm{Ba} / \mathrm{Eu}$ exhibits a regular increase with $[\mathrm{Fe} / \mathrm{H}] ;(2) \mathrm{La} / \mathrm{Eu}$ appears to lie within $\approx 0.3$ dex of the solar $r$-process value over $-3 \lesssim[\mathrm{Fe} / \mathrm{H}] \lesssim-1$ (with the exception of several data points); (3) $\mathrm{Nd} / \mathrm{Eu}$ shows a wide scatter with no identifiable trend; (4) Dy/Eu appears to lie within $\approx 0.3 \mathrm{dex}$ of the solar $r$-process value over $-3 \lesssim[\mathrm{Fe} / \mathrm{H}] \lesssim-1$ (with the exception of several data
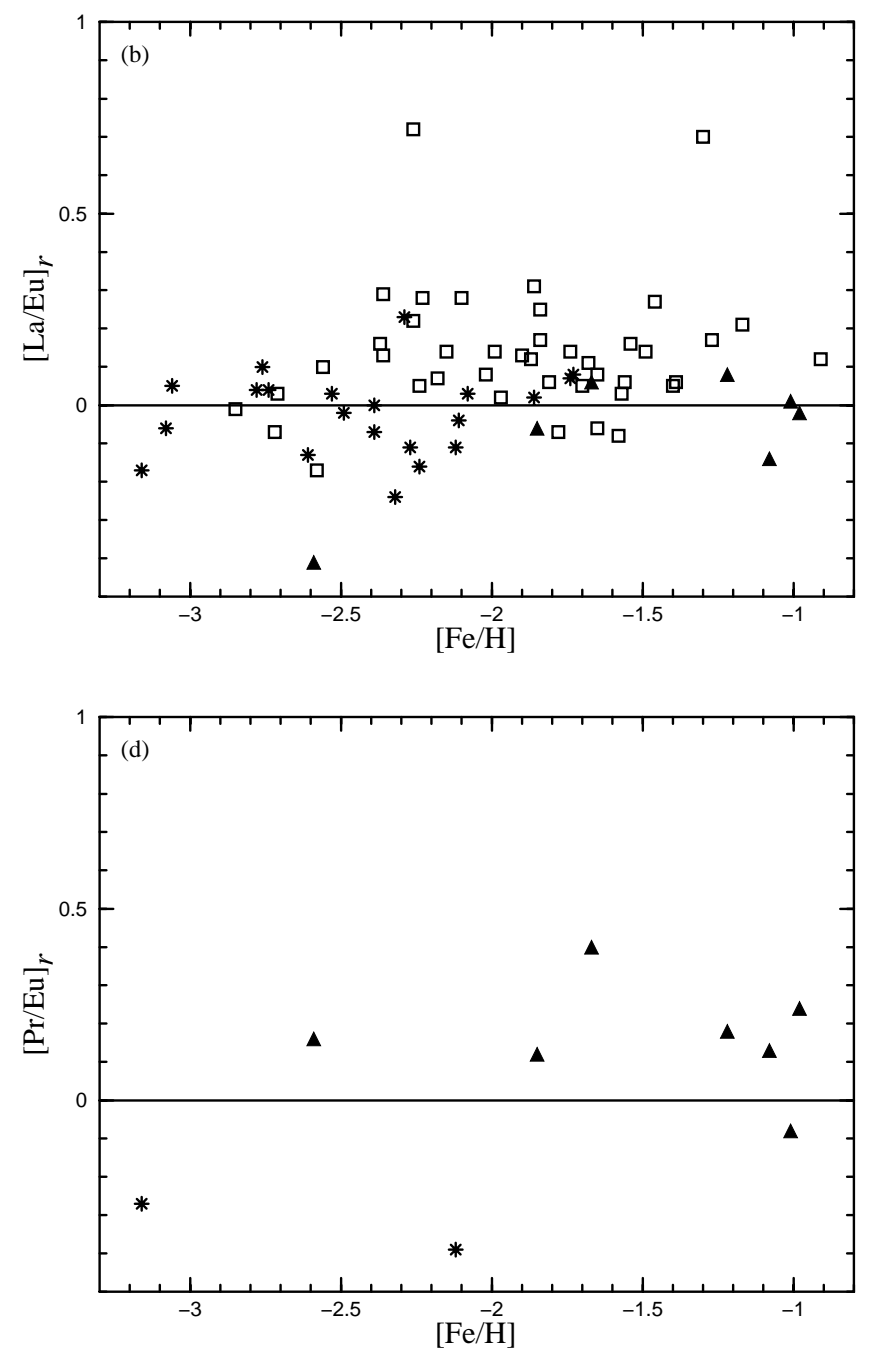

Fig. 3.-Data on $[\mathrm{E} / \mathrm{Eu}]_{r} \equiv \log (\mathrm{E} / \mathrm{Eu})-\log (\mathrm{E} / \mathrm{Eu})_{\odot, r}($ filled triangles: Gratton \& Sneden 1994; open squares: Burris et al. 2000; asterisks: Johnson \& Bolte 2001) shown as a function of $[\mathrm{Fe} / \mathrm{H}]$ for $(a) \mathrm{Ba},(b) \mathrm{La},(c) \mathrm{Ce},(d) \mathrm{Pr},(e) \mathrm{Nd},(f) \mathrm{Sm},(g) \mathrm{Gd}$, and $(h)$ Dy. Panel $(a)$ shows a regular increase of Ba/Eu above the standard solar $r$-process value of Arlandini et al. (1999) with increasing $[\mathrm{Fe} / \mathrm{H}]$. Panels $(b)$ and $(h)$ show that $\mathrm{La} / \mathrm{Eu}$ and $\mathrm{Dy} / \mathrm{Eu}$ are within $\approx 0.3 \mathrm{dex}$ of the standard solar $r$-process values (with the exception of several data points). Panels $(c),(d),(f)$, and $(g)$ show sparse data on $\mathrm{Ce} / \mathrm{Eu}, \mathrm{Pr} / \mathrm{Eu}, \mathrm{Sm} / \mathrm{Eu}$, and $\mathrm{Gd} / \mathrm{Eu}$ with no indication of any wide spread from the standard solar $r$-process values. Panel $(e)$ shows a wide scatter in $\mathrm{Nd} / \mathrm{Eu}$ with no identifiable trend. These data suggest that heavy $r$-elements from $\mathrm{La}$ and above come only from $H$ events and that s-process contributions are unimportant at $[\mathrm{Fe} / \mathrm{H}]<-1$. 

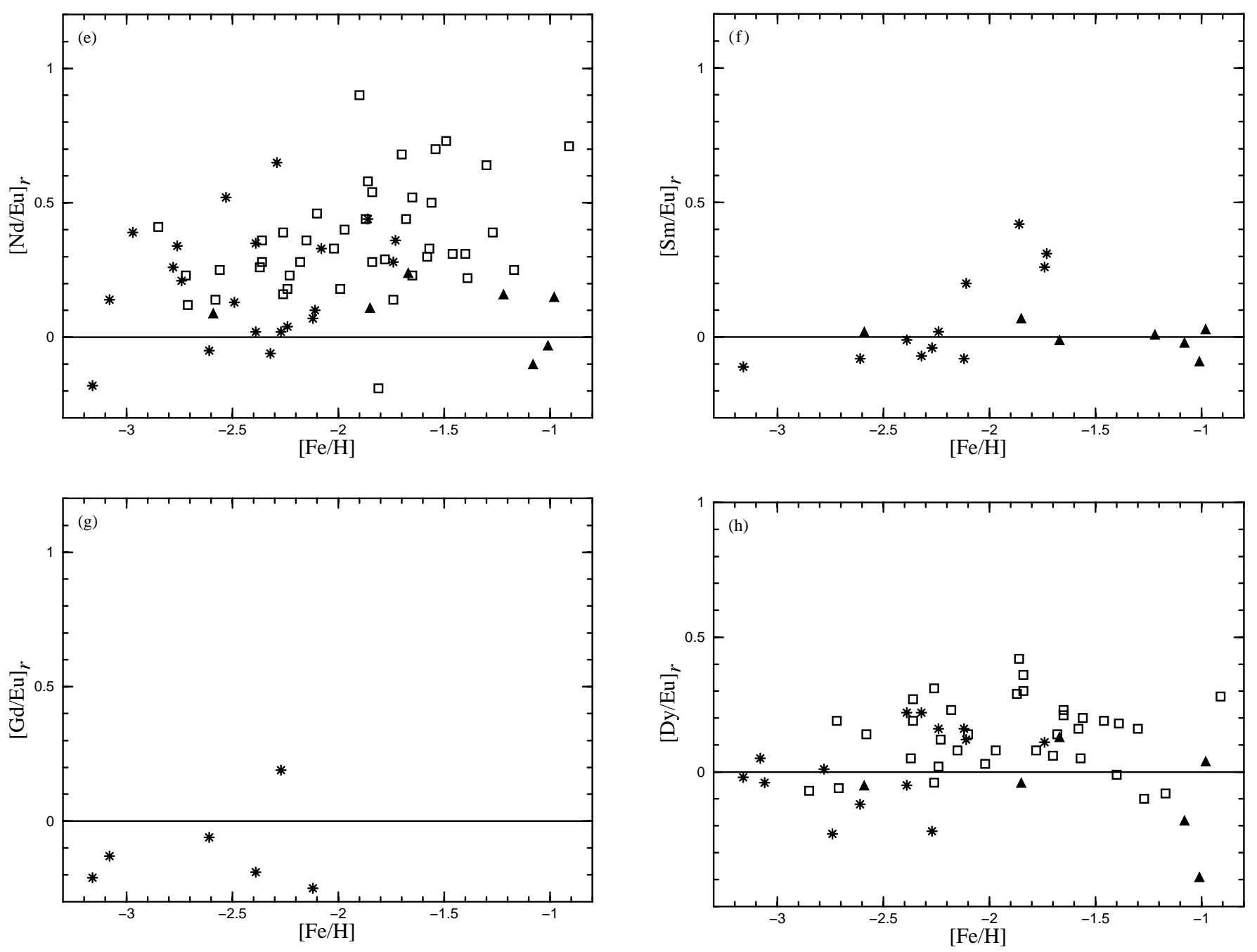

points); and (5) $\mathrm{Ce} / \mathrm{Eu}, \mathrm{Pr} / \mathrm{Eu}, \mathrm{Sm} / \mathrm{Eu}$, and $\mathrm{Gd} / \mathrm{Eu}$ have only sparse data with no indication of any wide spread from the solar $r$-process values. In general, all those elements above $\mathrm{Ba}$ (except for $\mathrm{Nd}$ ) appear to have ratios relative to Eu that are roughly constant. This demonstrates that they are to be associated exclusively with $H$ events, which is in accord with the model. We cannot explain the scatter in $\mathrm{Nd} / \mathrm{Eu}$. Further, we note that the observed values for $\mathrm{La} / \mathrm{Eu}, \mathrm{Ce} / \mathrm{Eu}$, $\operatorname{Pr} / \mathrm{Eu}, \mathrm{Sm} / \mathrm{Eu}, \mathrm{Gd} / \mathrm{Eu}$, and $\mathrm{Dy} / \mathrm{Eu}$ are close to the standard solar $r$-process values. Of these elements, $\mathrm{Sm}, \mathrm{Eu}, \mathrm{Gd}$, and Dy have predominant $r$-process contributions to their solar inventory $\left(\beta_{\odot, s} \ll 1\right.$; see Table 3$)$. However, La has $\beta_{\odot, s}(\mathrm{La})=0.62$ from stellar model calculations and $\beta_{\odot, s}(\mathrm{La})=0.83$ from the classical analysis (Arlandini et al. 1999). These $\beta_{\odot, s}(\mathrm{La})$ values correspond to a change in the solar $r$-component of La by a factor of 2.2. Thus, the present agreement between the observed $\mathrm{La} / \mathrm{Eu}$ and the corresponding solar $r$-process value from stellar model calculations of Arlandini et al. (1999) is surprising but encouraging. As La, Ce, and $\operatorname{Pr}$ have $\beta_{\odot, s} \gtrsim 0.5$ (see Table 3 ), it is clear that any significant s-process addition to the ISM would drastically shift their abundances away from the nominal solar $r$-process values. This is not observed. We conclude that the observational data appear to justify two basic assumptions of our model: the $H$ events dominate the production of heavy $r$-elements from $\mathrm{La}$ and above and

there is no significant $s$-process contribution to the ISM prior to $[\mathrm{Fe} / \mathrm{H}] \sim-1$. The new data also support and extend the earlier observational results that the $r$ abundance pattern above Ba in UMP stars is the same as that in the solar system (Sneden et al. 1996, 2000; Westin et al. 2000). The need for more precise observational data on $\mathrm{La}$ and above is evident.

Figure 3 shows that $\mathrm{Ba} / \mathrm{Eu}$ exhibits systematic deviation from a constant value with increasing $[\mathrm{Fe} / \mathrm{H}]$. Johnson \& Bolte (2001) have emphasized that the $\mathrm{Ba}$ abundance data may reflect uncertainties in stellar atmosphere models. These uncertainties may have caused the deviation for $\mathrm{Ba} / \mathrm{Eu}$ as well as the wide scatter in $\mathrm{Nd} / \mathrm{Eu}$ discussed above. However, the extent to which artifacts in stellar atmosphere models would affect the abundance analysis for Ba and other elements remains to be investigated. On the other hand, $\mathrm{Ba}$ is special in that it is closer to the boundary between light and heavy $r$-elements. This boundary has been considered to be at $A \sim 130$ (WBG96). It was also noted that any $r$-process model is expected to produce the abundance peak at $A \sim 130$ with some overflow to higher masses (Qian et al. 1998). In this case, some contributions may be expected at and above Ba from the $L$ events that are mainly responsible for the light $r$-elements. A test of such overflow would be shifts in the abundance ratios of other heavy $r$-elements to $\mathrm{Eu}$ with increasing $L$ contributions (i.e., 
increasing $[\mathrm{Fe} / \mathrm{H}])$. As discussed above, no such shifts have been observed for La (see Fig. 3). Thus, the overflow in $L$ events must stop below $\mathrm{La}(A=139)$. However, $\mathrm{Ba}$ is below La and may be liable to the influence of such overflow, so the deviation of $\mathrm{Ba} / \mathrm{Eu}$ from a constant value with increasing $[\mathrm{Fe} / \mathrm{H}]$ may be caused by the $L$ contributions to $\mathrm{Ba}$. This possibility will be discussed further in $\S 3.3$.

\subsection{Abundances of $\mathrm{Sr}, \mathrm{Y}, \mathrm{Zr}$, and $\mathrm{Ba}$ in UMP Stars}

The $P$-inventory and $H$ yields of $\mathrm{Sr}, \mathrm{Y}$, and $\mathrm{Zr}$ in Table 3 are calculated from data on HD 115444 and CS 22892-052 (both with $[\mathrm{Fe} / \mathrm{H}] \approx-3$ ). These results are used to calculate the $\mathrm{Sr}, \mathrm{Y}$, and $\mathrm{Zr}$ abundances in four other UMP stars in the sample of Burris et al. (2000). For example, BD $+58^{\circ} 1218$ has $\log \epsilon(\mathrm{Eu})=-2.00$ and $[\mathrm{Fe} / \mathrm{H}]=-2.72$ corresponding to $n_{H} \approx 3$ and $n_{L} \approx 0$. As its $[\mathrm{Fe} / \mathrm{H}]$ differs from $[\mathrm{Fe} / \mathrm{H}]_{P} \approx-3$ by $\delta \approx 0.28 \mathrm{dex}$, the corresponding $P$ components of $\mathrm{Sr}, \mathrm{Y}$, and $\mathrm{Zr}$ must be increased from the $\log \epsilon_{P}$ values in Table 3 by the same amount (see $\S 2.1$ ). The calculated $\mathrm{Sr}$ abundance for this star is $\log \epsilon_{\text {cal }}(\mathrm{Sr})=$ $\log \left[10^{\log \epsilon P(\mathrm{Sr})+\delta}+n_{H} \times 10^{\log \epsilon_{H}(\mathrm{Sr})}\right] \approx 0.43$. The values of $\log \epsilon_{\text {cal }}(\mathrm{Y}) \approx-0.71$ and $\log \epsilon_{\text {cal }}(\mathrm{Zr}) \approx 0.18$ are obtained similarly. These calculated abundances are within $\approx 0.2 \mathrm{dex}$
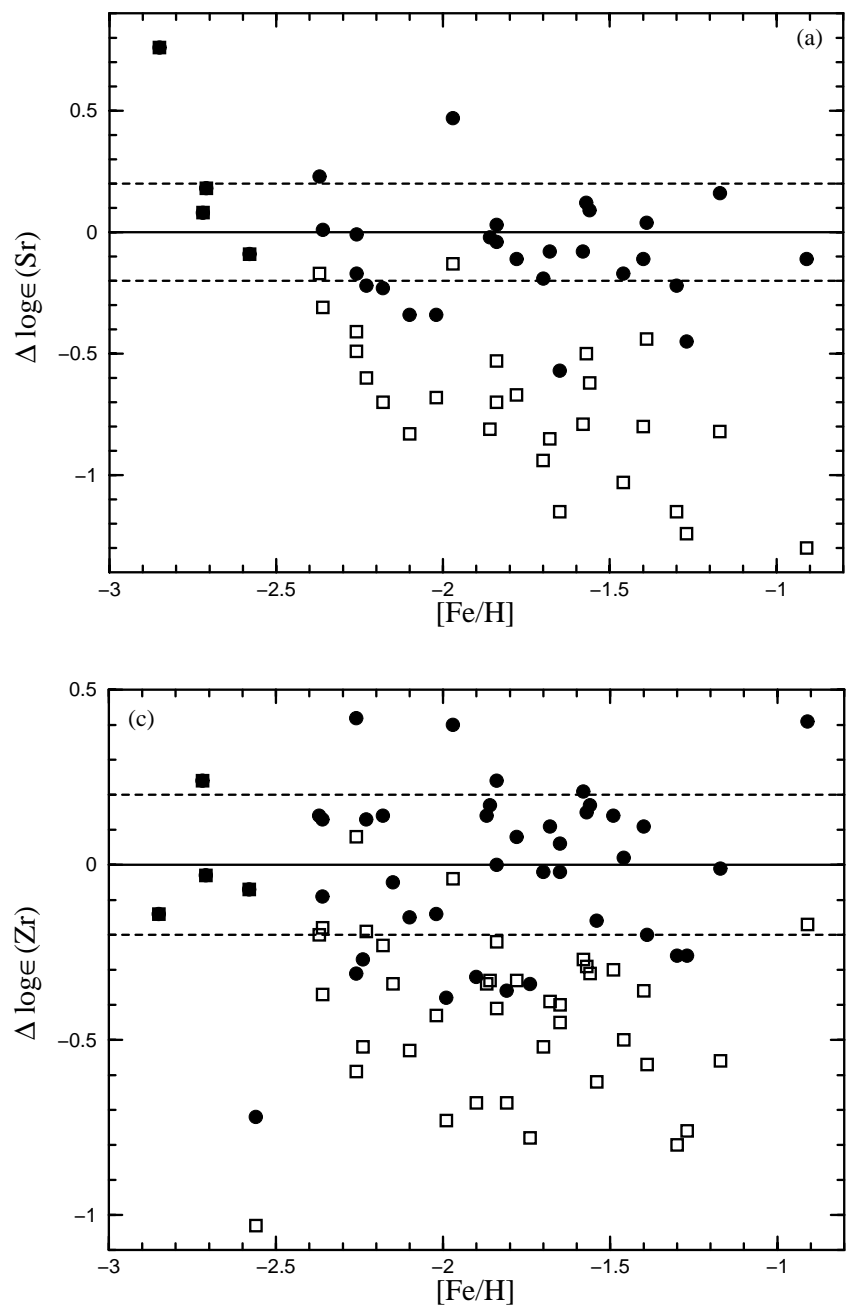

of the observational data $\log \epsilon_{\text {obs }}(\mathrm{Sr})=0.35, \log \epsilon_{\mathrm{obs}}(\mathrm{Y})=$ -0.58 , and $\log \epsilon_{\mathrm{obs}}(\mathrm{Zr})=-0.06$. The difference between the model results and data, $\Delta \log \epsilon(\mathrm{E}) \equiv \log \epsilon_{\mathrm{cal}}(\mathrm{E})$ $-\log \epsilon_{\mathrm{obs}}(\mathrm{E})$, is shown for the four UMP stars in the sample of Burris et al. (2000) in Figure 4 (filled squares). Except for $\mathrm{Sr}$ in HD 126587, $|\Delta \log \epsilon(\mathrm{E})| \lesssim 0.2$ dex for all other cases. We note that McWilliam et al. (1995) gave $\log \epsilon_{\mathrm{obs}}(\mathrm{Sr})=0.22$ for HD 126587, which is 0.62 dex higher than the value of Burris et al. (2000). Using the value of McWilliam et al. (1995) would bring the calculated Sr abundance of this star within 0.2 dex of the data.

With regard to $\mathrm{Ba}$ in UMP stars, we have calculated the abundances assuming pure $H$ contributions and using the observed $\log \epsilon(\mathrm{Eu})$ to determine the value of $n_{H}$. The calculated values are in very good agreement with the observational data (see Fig. 4, filled squares). There are two issues regarding the $\mathrm{Ba}$ data at $-4 \lesssim[\mathrm{Fe} / \mathrm{H}]<-2.5$ (see Fig. 1). The low $\mathrm{Ba}$ abundances at $-4 \lesssim[\mathrm{Fe} / \mathrm{H}]<-3$ compared with the $H$ yield of $\mathrm{Ba}$ (Fig. 1, dotted line) were attributed to very small admixtures of $H$ contributions into clouds having almost pure $P$-inventory composition. There is also a rapid rise of $\log \epsilon(\mathrm{Ba})$ at $-3 \lesssim[\mathrm{Fe} / \mathrm{H}]<-2.5$. The dotdashed line from the model (see $\S 3.3$ ) that describes the
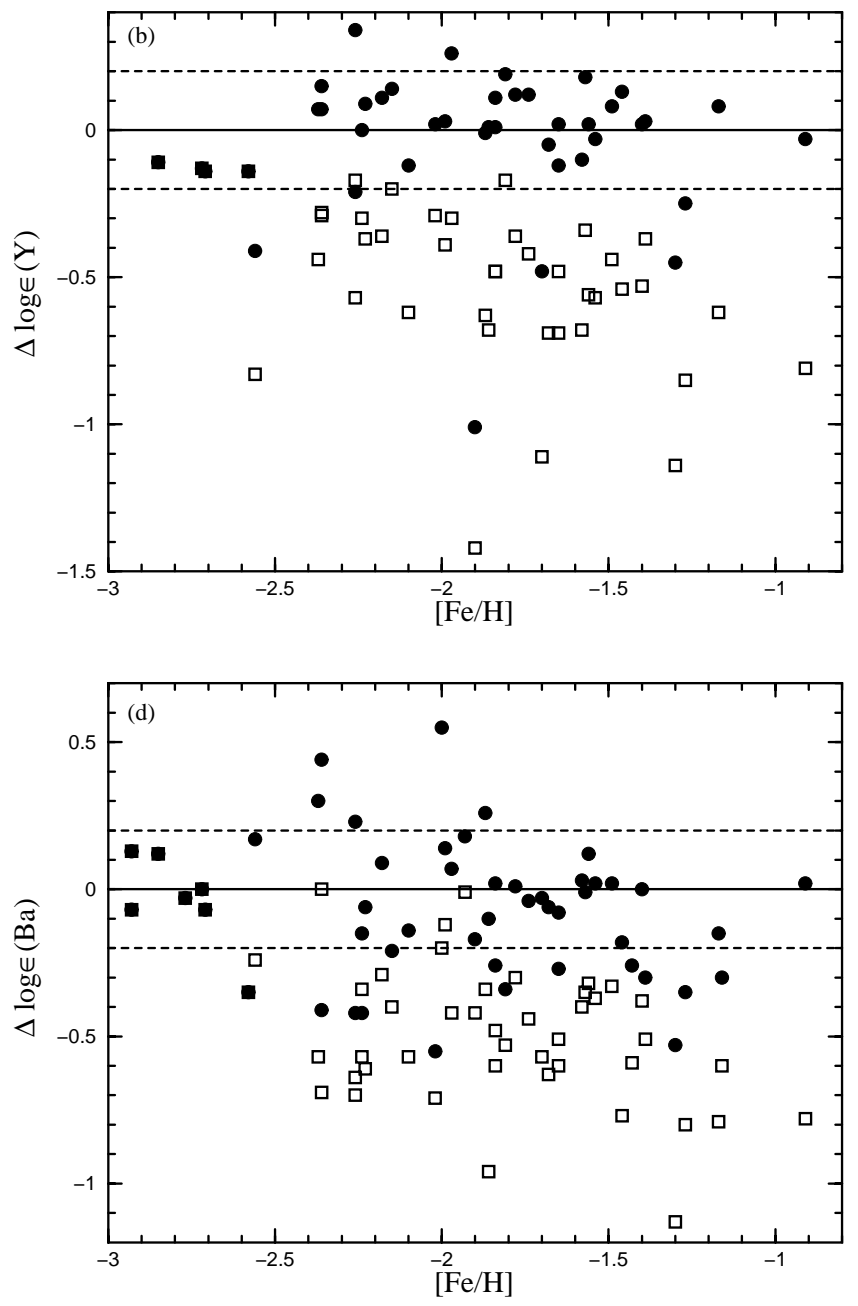

FIG. 4.-Difference between the abundances calculated from the model and the data of Burris et al. (2000), $\Delta \log \epsilon(\mathrm{E}) \equiv \log \epsilon_{\mathrm{cal}}(\mathrm{E})-\log \epsilon_{\mathrm{obs}}(\mathrm{E})$, shown as a function of $[\mathrm{Fe} / \mathrm{H}]$ for $(a) \mathrm{Sr},(b) \mathrm{Y},(c) \mathrm{Zr}$, and $(d) \mathrm{Ba}$. Open squares are for the standard solar $r$-abundances (col. [6] of Table 3) and filled circles for the corrected values proposed here (col. [9] of Table 3). Filled squares indicate UMP stars with $[\mathrm{Fe} / \mathrm{H}] \approx-3$. We estimate the uncertainties in the calculated abundances to be $\approx 0.2$ dex. These represent our generalized approximation of the uncertainties in the data for HD 115444 and CS $22892-052$ that are used to derive the model parameters. Burris et al. (2000) did not explicitly assign uncertainties to their data. 
trend of data at $[\mathrm{Fe} / \mathrm{H}]>-2.5$ where both $H$ and $L$ events occur is extended to $[\mathrm{Fe} / \mathrm{H}]=-4$ in Figure 1 . The deviation (by $\sim 1-2$ dex) of the data at $-3 \lesssim[\mathrm{Fe} / \mathrm{H}]<-2.5$ from the trend at $[\mathrm{Fe} / \mathrm{H}]>-2.5$ is evident. This is the wall of rapid rise described by WQ00 and was attributed to the onset of $H$ events with no Fe production. A problem exists as to how high this rise might be if it represents a sampling of the ISM. As the $H$ yield of $\mathrm{Ba}, \log \epsilon_{H}(\mathrm{Ba}) \approx-1.5$, is well established and the frequency of $H$ events is $\sim\left(10^{7} \mathrm{yr}\right)^{-1}$, it is clear that for a UMP star, $\log \epsilon(\mathrm{Ba})$ should not represent more $H$ events than, say, $n_{H} \sim 20$, corresponding to a period of $\sim 2 \times 10^{8} \mathrm{yr}$ during which an $L$ event should occur with very high probability. Observed values of $\log \epsilon(\mathrm{Ba})$ representing $n_{H} \gtrsim 30 \mathrm{H}$ events but with no concomitant increase in $[\mathrm{Fe} / \mathrm{H}]$, such as in the cases of CS 31082-001 (Fig. 1, crosses linked by a line) and CS 22892-052 (open circle), are then attributed to contamination by an SN II $H$ event in a binary and not to gross increases of $r$ abundances in the ISM from which the stars formed (QW01). Surface contamination may also apply to some data at $[\mathrm{Fe} / \mathrm{H}]>-2.5$ that lie far above the trend line in Figure 1. Clearly, chemical enrichments by surface contamination in binary systems cannot be ignored (see Preston \& Sneden 2001).

\subsection{Abundances of $\mathrm{Sr}, \mathrm{Y}, \mathrm{Zr}$, and $\mathrm{Ba}$ in MP stars}

Here we focus on the discussion of MP stars. The range of $-3<[\mathrm{Fe} / \mathrm{H}]<-1$ for MP stars is considered to result from addition of $\mathrm{Fe}$ from $L$ events to the $P$-inventory. In extending the discussion from UMP stars with no contributions from $L$ events to MP stars with such contributions, estimates of the $L$ yields play an essential role. The $L$ yields, $\log \epsilon_{L}(\mathrm{E})$, are calculated from the values of $\log \epsilon_{\odot, r}(\mathrm{E})$, $\log \epsilon_{P}(\mathrm{E})$, and $\log \epsilon_{H}(\mathrm{E})$ given in Table 3. The abundances of $\mathrm{Sr}, \mathrm{Y}$, and $\mathrm{Zr}$ are then calculated from the model by using the $P$-inventory and $H$ and $L$ yields together with the observed $\log \epsilon(\mathrm{Eu})$ and $[\mathrm{Fe} / \mathrm{H}]$ for the MP stars in the sample of Burris et al. (2000). For example, HD 122956 has $\log \epsilon(\mathrm{Eu})=-0.72$ and $[\mathrm{Fe} / \mathrm{H}]=-1.78$ corresponding to $n_{H} \approx 58$ and $n_{L} \approx 5$. The calculated $\mathrm{Sr}$ abundance for this star is $\log \epsilon_{\mathrm{cal}}(\mathrm{Sr})=\log \left[10^{\log \epsilon P(\mathrm{Sr})}+n_{H} \times 10^{\log \epsilon_{H}(\mathrm{Sr})}+n_{L}\right.$ $\left.\times 10^{\log \epsilon_{L}(\mathrm{Sr})}\right] \approx 0.63$. The values of $\log \epsilon_{\text {cal }}(\mathrm{Y}) \approx-0.08$ and $\log \epsilon_{\text {cal }}(\mathrm{Zr}) \approx 0.65$ are obtained similarly. By comparison, the observed values are $\log \epsilon_{\mathrm{obs}}(\mathrm{Sr})=1.30$, $\log \epsilon_{\mathrm{obs}}(\mathrm{Y})=0.28$, and $\log \epsilon_{\mathrm{obs}}(\mathrm{Zr})=0.98$. Thus, the calculated abundances (especially for $\mathrm{Sr}$ ) significantly differ from the data. The difference between the model results and data, $\Delta \log \epsilon(\mathrm{E}) \equiv \log \epsilon_{\mathrm{cal}}(\mathrm{E})-\log \epsilon_{\mathrm{obs}}(\mathrm{E})$, is shown as a function of $[\mathrm{Fe} / \mathrm{H}]$ for the MP stars in the sample of Burris et al. (2000) in Figure 4 (open squares). It can be seen that the model systematically underestimates $\mathrm{Sr}, \mathrm{Y}$, and $\mathrm{Zr}$ abundances at $-2.5 \lesssim[\mathrm{Fe} / \mathrm{H}]<-1$. As these metallicities correspond to increasing contributions from $L$ events, we consider that this systematic deviation is possibly caused by errors in the $L$ yields of $\mathrm{Sr}, \mathrm{Y}$, and $\mathrm{Zr}$ as determined from the solar $r$-inventory.

Note that the solar $r$-abundance of $\mathrm{Sr}$ assigned by Arlandini et al. (1999) is saturated by the contributions from $n_{H}^{\odot}=10^{3} \mathrm{H}$ events and thus does not allow any $L$ contributions to Sr. However, the solar $r$-abundances of $\mathrm{Y}$ and $\mathrm{Zr}$ assigned by the same authors allow substantial $L$ contributions to these two elements (see Table 3). As Sr, Y, and Zr are close in atomic mass, it is rather difficult to explain why $L$ events can produce $\mathrm{Y}$ and $\mathrm{Zr}$ but no Sr. It seems more likely that the standard solar $r$-abundances of $\mathrm{Sr}, \mathrm{Y}$, and $\mathrm{Zr}$ are too uncertain to give reliable $L$ yields for these elements. As discussed in $\S 1.5$, the solar $r$-process fraction $\left[1-\beta_{\odot, s}(\mathrm{E})\right]$ for element $\mathrm{E}$ can have quite large errors when $\beta_{\odot, s}(\mathrm{E})$ is close to unity as for $\mathrm{Sr}, \mathrm{Y}$, and $\mathrm{Zr}$ (see Table 3 ). We now consider that the solar $r$-inventory of Sr, Y, and $\mathrm{Zr}$ given by Arlandini et al. (1999) and adopted above may be increased by factors of $6.3,3.5$, and 3 , respectively, to obtain a "corrected" solar $r$-inventory $\log \epsilon_{\odot, r}^{\text {corr }}(E)$ and the corresponding $\log \epsilon_{L}^{\text {corr }}(E)$ values in Table 3. With these $\log \epsilon_{L}^{\text {corr }}(\mathrm{E})$ values, the model results on $\mathrm{Sr}, \mathrm{Y}$, and $\mathrm{Zr}$ abundances at $-2.5 \lesssim[\mathrm{Fe} / \mathrm{H}]<-1$ are generally in quite good agreement with data (see Fig. 4, filled circles). The problems and issues that are raised by the hypothesized shifts in $\beta_{\odot, s}(\mathrm{E})$ for $\mathrm{Sr}, \mathrm{Y}$, and $\mathrm{Zr}$ will be discussed in $\S 4$. It has been well known for some time that the abundances of Sr, Y, and $\mathrm{Zr}$ in UMP and MP stars cannot be explained by any standard nucleosynthetic models (M. Busso \& R. Gallino 2001, private communication). We note that the $P$ inventory, $H$ yields, and corrected $L$ yields of these elements given here follow approximately the same relative production pattern with a significant dip at $Y$. This is not the case if the standard $\beta_{\odot, s}$ values for $\mathrm{Sr}, \mathrm{Y}$, and $\mathrm{Zr}$ are used.

If we assign $r$-process production of $\mathrm{Ba}$ exclusively to $H$ events, the $\mathrm{Ba}$ abundance in an MP star can be calculated as $\log \epsilon_{\text {cal }}(\mathrm{Ba})=\log \epsilon_{H}(\mathrm{Ba})+\log n_{H}$, where $\log \epsilon_{H}(\mathrm{Ba})$ can be obtained from the solar $r$-inventory of $\mathrm{Ba}$ for $n_{H}^{\odot}=10^{3}$ or from the data on CS 22892-052 with essentially the same result (see $\S 2.1$ and Table 3). The difference between the calculated results and data of Burris et al. (2000), $\Delta \log \epsilon(\mathrm{Ba}) \equiv \log \epsilon_{\mathrm{cal}}(\mathrm{Ba})-\log \epsilon_{\mathrm{obs}}(\mathrm{Ba})$, is shown as a function of $[\mathrm{Fe} / \mathrm{H}]$ in Figure 4 (open squares). Note that $\Delta \log \epsilon(\mathrm{Ba})$ lies significantly below zero at $-2.5 \lesssim[\mathrm{Fe} / \mathrm{H}]<-1$. This is the same as found for $\mathrm{Sr}, \mathrm{Y}$, and $\mathrm{Zr}$. As indicated above, this result follows whether we calculate $\log \epsilon_{H}(\mathrm{Ba})$ by using the data on a UMP star or by attributing the standard solar $r$-inventory of Ba from Arlandini et al. (1999) to $n_{H}^{\odot}=10^{3} \mathrm{H}$ events. If we consider that the solar $r$-inventory of $\mathrm{Ba}$ assumed above is too small and that $L$ events contribute some $\mathrm{Ba}$, we may find a change that could eliminate the discrepancy. Using $\log \epsilon_{H}(\mathrm{Ba}) \approx$ -1.57 calculated from the data on CS 22892-052 (see $\$ 2.1$ ) and increasing the solar $r$-inventory of Ba by a factor of 2 from the value given by Arlandini et al. (1999), we obtain $\log \epsilon_{L}^{\text {corr }}(\mathrm{Ba}) \approx-0.47$ (see eq. [17]). We then recalculate $\log \epsilon_{\mathrm{cal}}(\mathrm{Ba})$ from equation (4) by including the contributions to $\mathrm{Ba}$ from $L$ events. The recalculated $\Delta \log \epsilon(\mathrm{Ba})$ is shown in Figure 4 (filled circles). It can be seen that the model results with Ba contributions from $L$ events represent data quite well over $-2.5 \lesssim[\mathrm{Fe} / \mathrm{H}]<-1$. This also accounts for the trend of $\mathrm{Ba} / \mathrm{Eu}$ (see $\S 3.1$ and Fig. 3) found by Burris et al. (2000) and Johnson \& Bolte (2001): the shift in Ba/Eu with $[\mathrm{Fe} / \mathrm{H}]$ is caused by the increasing contributions to $\mathrm{Ba}$ from $L$ events that do not produce Eu. The mean trend for evolution of $\mathrm{Ba}$ abundance relative to $\mathrm{Fe}$ at $[\mathrm{Fe} / \mathrm{H}]>-2.5$ can be calculated from the values of $\log \epsilon_{H}(\mathrm{Ba}), \log \epsilon_{L}^{\text {corr }}(\mathrm{Ba})$, and $\log \epsilon_{L}(\mathrm{Fe})$ by assuming $n_{H} / n_{L}=10$. This is shown as the dot-dashed line in Figure 1.

As an independent test, we may calculate $\log \epsilon_{L}(\mathrm{Ba})$ from data on HD 126238 (Gratton \& Sneden 1994), which is not in the sample of Burris et al. (2000). In this calculation the part of the solar Fe inventory contributed by $\mathrm{SNe}$ II is used to determine the $L$ yield of $\mathrm{Fe}$, and hence $n_{L}$, but the solar $r$-inventory of $\mathrm{Ba}$ is not used. The value of $\log \epsilon_{L}(\mathrm{Ba})$ is 
calculated from the value of $\log \epsilon_{H}(\mathrm{Ba})$ (obtained from the data on CS 22892-052) and the observed value of $\log \epsilon(\mathrm{Ba})$ in HD 126238 together with the values of $n_{H}$ and $n_{L}$ for this star. The observed values of $\log \epsilon(\mathrm{Eu})=-0.87$ and $[\mathrm{Fe} / \mathrm{H}]=-1.67$ for $\mathrm{HD} 126238$ correspond to $n_{H} \approx 41$ and $n_{L} \approx 6$. Using $\log \epsilon_{H}(\mathrm{Ba}) \approx-1.57$, we obtain $\log \epsilon_{L}(\mathrm{Ba}) \approx-0.46$ from the observed value of $\log \epsilon(\mathrm{Ba})=0.50$ for HD 126238. This is in excellent agreement with the above value of $\log \epsilon_{L}^{\text {corr }}(B a) \approx-0.47$. It thus appears that the value of $\log \epsilon_{L}(\mathrm{Ba})$ directly determined from data on a single MP star not in the sample of Burris et al. (2000) without using the solar $r$-inventory of $\mathrm{Ba}$ is in accord with the value obtained by changing this inventory to fit the data of Burris et al. (2000) on a large sample of MP stars. We consider the above direct calculation and the problem of fitting the $\mathrm{Ba}$ data as some evidence indicating that the standard solar $r$-inventory of $\mathrm{Ba}$ may require revision. The required change in this inventory by a factor of 2 is not within the $1 \sigma$ error band for $\left[1-\beta_{\odot, s}(\mathrm{Ba})\right]$ given by Arlandini et al. (1999) but corresponds to a change of only $23 \%$ from their assigned value of $\beta_{\odot, s}(\mathrm{Ba})=0.81$ to the corrected value of $\beta_{\odot, s}^{\text {corr }}(\mathrm{Ba})=0.62$.
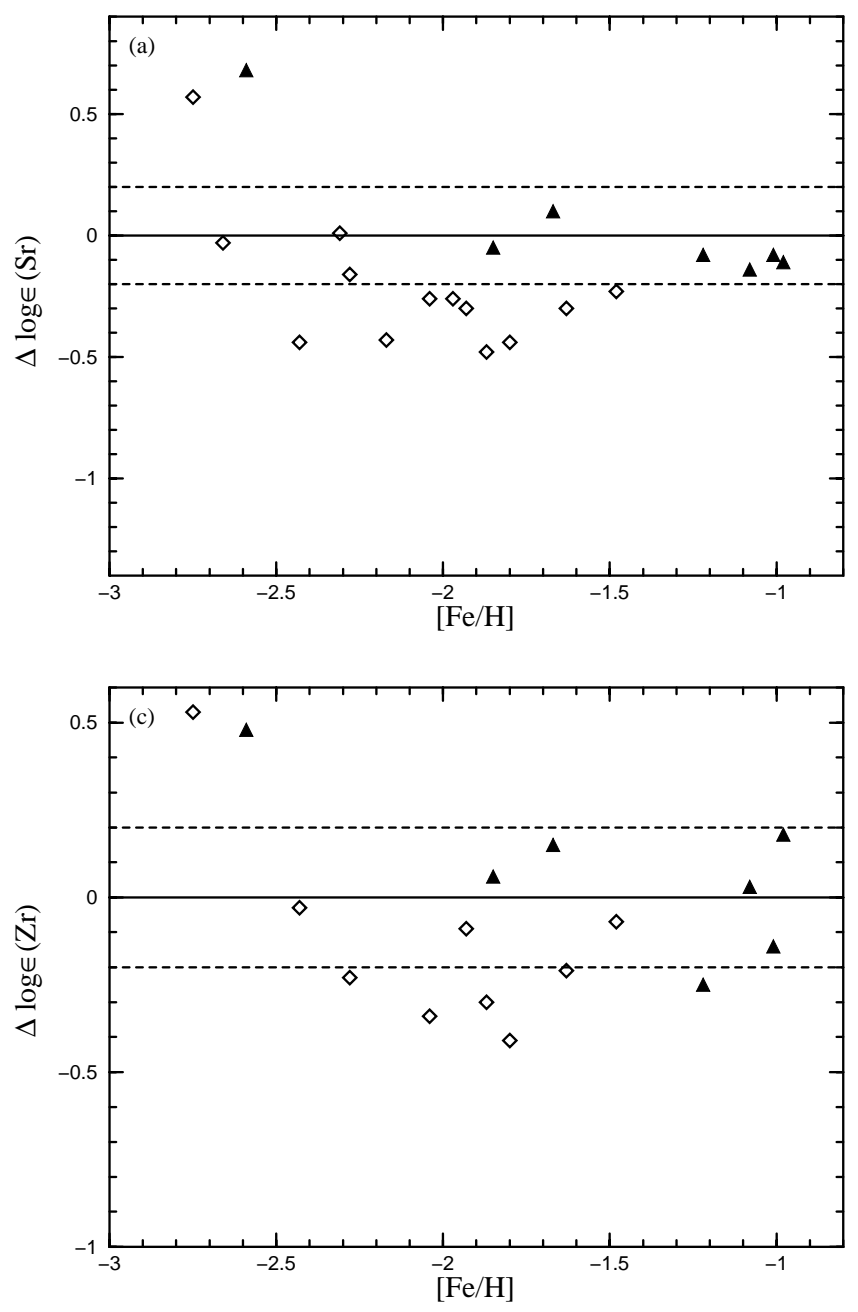

To test further the model with corrected solar $r$-inventory of $\mathrm{Sr}, \mathrm{Y}, \mathrm{Zr}$, and $\mathrm{Ba}$, we apply it to the data sets of Magain (1989) and Gratton \& Sneden (1994) that are distinct from the data set of Burris et al. (2000). The difference between the model results and data, $\Delta \log \epsilon(\mathrm{E}) \equiv \log \epsilon_{\mathrm{cal}}(\mathrm{E})$ $-\log \epsilon_{\mathrm{obs}}(\mathrm{E})$, is shown for $\mathrm{Sr}, \mathrm{Y}, \mathrm{Zr}$, and $\mathrm{Ba}$ as a function of $[\mathrm{Fe} / \mathrm{H}]$ in Figure 5 . It can be seen that by using the corrections made to the solar $r$-inventory for these elements based on the data set of Burris et al. (2000), the model describes the data sets of Magain (1989) and Gratton \& Sneden (1994) equally well $(|\Delta \log \epsilon(\mathrm{E})| \lesssim 0.2$ dex in most cases). Thus, we consider that the model presented above at least represents a very good parametric fit to the observational data on UMP and MP stars from several groups. It also seems possible that the model has deeper implications for $s$ process and $r$-process nucleosynthesis models and for the chemical evolution of the Galaxy.

\section{DISCUSSION AND CONCLUSIONS}

We have developed the consequences of a phenomenological model for the abundances of elements in the ISM for $[\mathrm{Fe} / \mathrm{H}]<-1$. This model assumes three components of
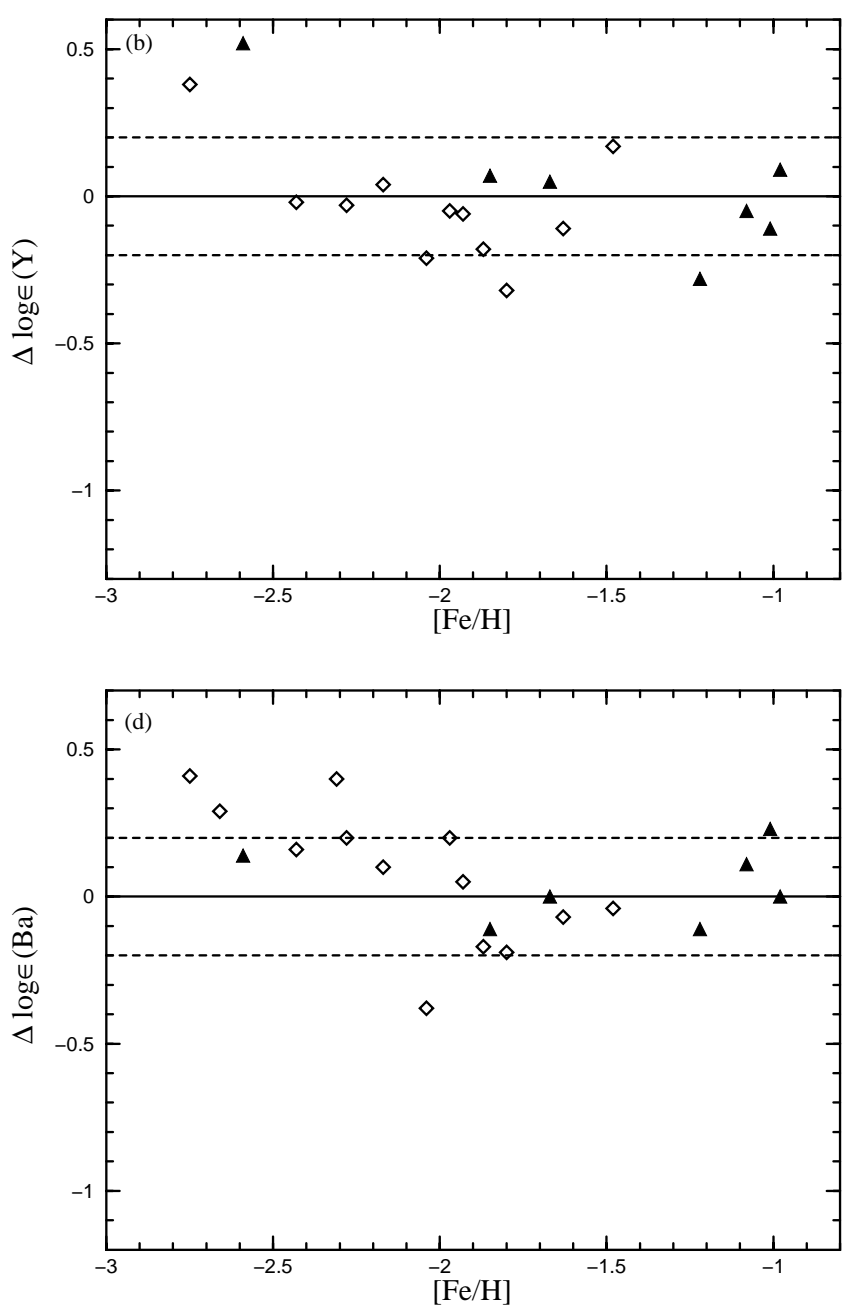

FIG. 5.-Difference between the abundances calculated from the model with the corrected solar $r$-inventory and the data of Magain (1989; open diamonds) and Gratton \& Sneden (1994; filled triangles), $\Delta \log \epsilon(\mathrm{E}) \equiv \log \epsilon_{\mathrm{cal}}(\mathrm{E})-\log \epsilon_{\mathrm{obs}}(\mathrm{E})$, shown as a function of $[\mathrm{Fe} / \mathrm{H}]$ for $(a) \mathrm{Sr},(b) \mathrm{Y},(c) \mathrm{Zr}$, and $(d) \mathrm{Ba}$. Note that by using the corrected solar $r$-inventory for these elements based on the data set of Burris et al. (2000), the model describes the two different data sets shown in this figure equally well. We estimate the uncertainties in the calculated abundances to be $\approx 0.2$ dex. These are comparable to the uncertainties in the data of Magain (1989) and Gratton \& Sneden (1994). In (c), the data for the open diamonds at $[\mathrm{Fe} / \mathrm{H}]=-2.75$ and -2.43 are taken from Zhao \& Magain (1991). 
nucleosynthetic contributions to $r$-process related elements. One component is contributed by the first generations of very massive ( $\gtrsim 100 M_{\odot}$ ) stars formed after the big bang. These stars provide an initial or prompt inventory of $\mathrm{Fe}$ and associated elements corresponding to $[\mathrm{Fe} / \mathrm{H}]_{P} \approx-3$. This $P$-inventory is distinctive and contains no heavy $r$ elements (with $A>130$ ). Once the $P$-inventory is established, normal stellar populations with masses of $\sim 1-60$ $M_{\odot}$, which include the progenitors for two types of SNe II, begin to form at $[\mathrm{Fe} / \mathrm{H}] \approx-3$. The two types of SNe II correspond to the other two components of the model. They are the high-frequency $H$ events that produce mainly heavy $r$-elements but no $\mathrm{Fe}$ and the low-frequency $L$ events that produce mainly light $r$-elements (with $A \lesssim 130$ ) and Fe. The composition of the $P$-inventory and the yield templates of $H$ and $L$ events are assumed to be fixed and distinct from each other. This allows the deconvolution of observational data to establish quantitatively the $P$-inventory and $H$ and $L$ yields. The basic input for this procedure is the observational data on two UMP stars and the solar $r$ abundances.

The element Eu commonly observed in UMP and MP stars is essentially a pure $r$-element. As Eu and all the other heavy $r$-elements above $\mathrm{Ba}$ are attributed exclusively to $H$ events, it is convenient to use the observed $\mathrm{Eu}$ abundance in a UMP or MP star to identify the $H$ contributions to the abundances in the star (any other heavy $r$-element above $\mathrm{Ba}$ can also be used for this purpose). The $H$ yield of $\mathrm{Eu}$ is calculated from its solar $r$-abundance by using the number of contributing $H$ events for the solar inventory, which can be estimated from the frequency of these events. The number of contributing $H$ events for a UMP or MP star can then be obtained from the observed $\mathrm{Eu}$ abundance in the star by using the $H$ yield of Eu. For UMP stars with $[\mathrm{Fe} / \mathrm{H}] \approx[\mathrm{Fe} / \mathrm{H}]_{P} \approx-3$, their abundances only consist of $P$ and $H$ contributions. By using the data on two UMP stars with $[\mathrm{Fe} / \mathrm{H}] \approx-3$ but with different Eu abundances, the $P$-inventory and $H$ yields of $\mathrm{Sr}, \mathrm{Y}$, and $\mathrm{Zr}$ are simultaneously obtained. The data on a third UMP star give essentially the same results for the $P$-inventory when corrected to the same $[\mathrm{Fe} / \mathrm{H}]_{P}$ value (see $\S 2.1$ ). These results are used in the model to calculate the $\mathrm{Sr}, \mathrm{Y}$, and $\mathrm{Zr}$ abundances in other UMP stars. The calculated abundances are shown to be in very good agreement with the observational data (see Fig. 4, filled squares). The $P$-inventory represents the sum of contributions from the first generations of very massive stars. We consider that the $P$-inventory of $\mathrm{Sr}, \mathrm{Y}$, and $\mathrm{Zr}$ reported here is reliable and may serve as a guide in evaluating models of nucleosynthesis for these stars.

The $H$ yields of heavy $r$-elements above Ba other than $\mathrm{Eu}$ can be calculated from the solar $r$-abundances as in the case of Eu or from the observed abundances in a UMP star by using the observed Eu abundance to obtain the number of contributing $H$ events for the star. These two approaches give effectively the same results as the $r$-abundance pattern above Ba observed in UMP stars is the same as that in the solar system. For MP stars with $-3<[\mathrm{Fe} / \mathrm{H}]<-1$, their $\mathrm{Fe}$ abundances reflect contributions from $L$ events. In general, the abundances in these stars are governed by $P, H$, and $L$ contributions. For the heavy $r$-elements above $\mathrm{Ba}$ that only have $H$ contributions in our model, the abundance ratios of the other heavy $r$-elements to $\mathrm{Eu}$ in MP stars are close to the solar $r$-process values assigned by Arlandini et al. (1999) over a wide range in $[\mathrm{Fe} / \mathrm{H}]$ (see Fig.
3). For other elements that have $L$ contributions, their $L$ yields are estimated from their solar $r$-abundances, $P$ inventory, and $H$ yields by using the numbers of contributing $H$ and $L$ events for the solar inventory. The abundances of these elements in an MP star can then be calculated from the three-component model by using the observed $\mathrm{Eu}$ and $\mathrm{Fe}$ abundances to obtain the numbers of contributing $H$ and $L$ events for the star. By comparing the model results with the observational data on $\sim 30-40 \mathrm{MP}$ stars in the sample of Burris et al. (2000), it is found that to match the $\mathrm{Sr}, \mathrm{Y}, \mathrm{Zr}$, and $\mathrm{Ba}$ data requires major changes in the solar $r$-abundances of these elements assigned by Arlandini et al. (1999) (see Table 3 and Fig. 4). The model with a set of corrected solar $r$-abundances for these elements determined by fitting the data set of Burris et al. (2000) is shown to describe the two different data sets of Magain (1989) and Gratton \& Sneden (1994) equally well over a wide range in $[\mathrm{Fe} / \mathrm{H}]$ (see Fig. 5). All of the data sets on Ba exhibit increases of $\mathrm{Ba} / \mathrm{Eu}$ above the standard solar $r$-process value with increasing $[\mathrm{Fe} / \mathrm{H}]$. This can be explained by the increasing contributions to $\mathrm{Ba}$ from $L$ events that do not produce $\mathrm{Eu}$ based on the model with the corrected solar $r$-abundance of $\mathrm{Ba}$.

In summary, by using the $P$-inventory, $H$ yields, and $L$ yields (see Table 3 and Fig. 3) that are obtained from the data on two UMP stars, the corrected solar $r$-abundances for $\mathrm{Sr}, \mathrm{Y}, \mathrm{Zr}$, and $\mathrm{Ba}$, and the standard solar $r$-abundances of Arlandini et al. (1999) for other elements, it is possible to calculate the abundances of a large number of elements in a UMP or MP star simply from the observed $\mathrm{Eu}$ and $\mathrm{Fe}$ abundances in the star over the range $-3 \lesssim[\mathrm{Fe} / \mathrm{H}]<-1$.

The fundamental question arises as to whether the corrected solar $r$-abundances for $\mathrm{Sr}, \mathrm{Y}, \mathrm{Zr}$, and $\mathrm{Ba}$ can be justified in terms of nuclear astrophysical considerations. All the other elements do not seem to require a significant shift in their solar $r$-inventory. It is shown that the model is robust insofar as the relative yield patterns for $H$ and $L$ events are fixed. Changes in the absolute yields (but not the relative yields) do not change the patterns but only the numbers of contributing $H$ and $L$ events for a star. The requirement for a large shift in the solar $r$-inventory for $\mathrm{Sr}$, $\mathrm{Y}, \mathrm{Zr}$, and $\mathrm{Ba}$ then remains unchanged in this case. With regard to contributions from other sources, we note that the observational data on $\mathrm{La}, \mathrm{Ce}$, and $\mathrm{Pr}$ with solar s-process fractions of $\beta_{\odot, s} \gtrsim 0.5$ show no evidence of significant $s$ process contributions to the ISM at $[\mathrm{Fe} / \mathrm{H}]<-1$. Thus, possible s-process additions to the ISM from AGB stars are unimportant at $[\mathrm{Fe} / \mathrm{H}]<-1$ and cannot affect the required changes in the solar $r$-inventory for $\mathrm{Sr}, \mathrm{Y}, \mathrm{Zr}$, and $\mathrm{Ba}$. We note that extremely high s-process enrichment has been observed in LP 625-44 with $[\mathrm{Fe} / \mathrm{H}]=-2.7$ (Aoki et al. 2000). However, this s-process enrichment does not reflect the composition of the ISM at $[\mathrm{Fe} / \mathrm{H}]=-2.7$ from which the star formed but is due to surface contamination by mass transfer from the binary companion of the star. Further, the observed $[\mathrm{Fe} / \mathrm{H}]$ in the star only indicates the formation time of the binary but does not correspond to the much later occurrence of $s$-processing in the AGB companion.

It is possible that some other aspects of the model not considered in the preceding discussion cause the disagreement between the model results and observational data for $\mathrm{Sr}, \mathrm{Y}, \mathrm{Zr}$, and $\mathrm{Ba}$ when the "standard" solar $r$-inventory for these elements is used. For example, the relative yields of 
the pertinent elements may not be fixed as assumed but may vary from one $H$ or $L$ event to another. In this case, the $H$ and $L$ contributions to the abundances in a star cannot be identified simply by using the observed abundances of Eu and $\mathrm{Fe}$ in the star. The problem of abundances in UMP and MP stars would then require a totally different approach. Unless confronted with new data that indicate otherwise, we adhere to the basic assumption of constant relative yields for $H$ or $L$ events and consider the following obvious alternatives to explain the disagreement referred to above: (1) there are some systematic shifts in the observational data provided by several groups for $\mathrm{Sr}, \mathrm{Y}, \mathrm{Zr}$, and $\mathrm{Ba}$ as a function of $[\mathrm{Fe} / \mathrm{H}]$, perhaps due to uncertainties in the stellar atmosphere models as suggested by Johnson \& Bolte (2001) for $\mathrm{Ba}$; or (2) there are intrinsic errors in the solar $r$ inventory for $\mathrm{Sr}, \mathrm{Y}, \mathrm{Zr}$, and $\mathrm{Ba}$.

The observed $\mathrm{Ba} / \mathrm{Eu}$ shows a regular increase above the standard solar $r$-process value with increasing $[\mathrm{Fe} / \mathrm{H}]$. However, the $H$ yield of $\mathrm{Ba}$ calculated from the data on a UMP star is in exact agreement with its solar $r$-abundance assigned by Arlandini et al. (1999) if there are no $L$ contributions to $\mathrm{Ba}$. This suggests that the observed shifts in $\mathrm{Ba} / \mathrm{Eu}$ with increasing $[\mathrm{Fe} / \mathrm{H}]$ are the result of an artifact in abundance analysis that also depends on $[\mathrm{Fe} / \mathrm{H}]$. We find it difficult to understand how artifacts in analysis of the observational data would cause large shifts in abundance only for $\mathrm{Ba}$ without having large effects on other elements. If the $\mathrm{Ba}$ problem is due to such an artifact, then the remaining inconsistency for $\mathrm{Sr}, \mathrm{Y}$, and $\mathrm{Zr}$ may be accounted for by the acceptable $s$-process uncertainties and hence errors in the solar $r$-inventory for these elements. We emphasize that for $\mathrm{Sr}, \mathrm{Y}$, and $\mathrm{Zr}$, the $\beta_{\odot, s}$ values calculated from s-process models are all close to unity and lie in a region where serious uncertainties or errors may exist as has long been recognized by experts in s-process modeling. If the $\mathrm{Ba}$ problem is not due to artifacts in the abundance analysis, then the matter is not simply clarified without changing the standard solar $r$-abundance of $\mathrm{Ba}$. The case of $\mathrm{Ba}$ is similar to that of $\mathrm{Sr}, \mathrm{Y}$, and $\mathrm{Zr}$ as $\beta_{\odot, s}(\mathrm{Ba})$ is also close to unity. However, $\mathrm{Ba}$ lies in a region where the main s-component is dominant and is subject to more constraints than $\mathrm{Sr}, \mathrm{Y}$, and $\mathrm{Zr}$. At present, the solution to this problem is not evident. However, there is a legitimate basis to consider that the solar $r$-component obtained by subtracting the $s$ component is subject to real uncertainties for elements such as $\mathrm{Sr}, \mathrm{Y}, \mathrm{Zr}$, and $\mathrm{Ba}$ with predominant s-process contribu- tions to their solar inventory. The solar s-component used here corresponds to an average of the $s$-process yields from models of 1.5 and $3 M_{\odot}$ AGB stars with $Z=0.5 Z_{\odot}$ that best reproduces the classical main $s$-component (Arlandini et al. 1999). In fact, the solar s-component is the integral of long-term Galactic chemical evolution starting with lowmetallicity AGB stars. Researchers in the field are fully aware of this matter. The solar $r$-component derived from the subtraction procedure inherits all the uncertainties in the $s$-component calculated from models. A reliable ab initio calculation of the solar $r$-component based on models of SNe II and Galactic chemical evolution remains to be pursued. Critical attention to the investigation of $s$-process and $r$-process nucleosynthesis indicated above is required to sort out the problems.

In any case, based on the observations of UMP stars, the $P$-inventory produced by the first generations of very massive stars and yields of SN II $H$ events appear to be well established. This constitutes a prediction of what nucleosynthetic models for very massive zero-metallicity stars and for the common SNe II should produce. By using the threecomponent model with corrected solar $r$-abundances for $\mathrm{Sr}$, $\mathrm{Y}, \mathrm{Zr}$, and $\mathrm{Ba}$ and the standard values for other elements, the abundances of a large number of elements in stars with $-3 \lesssim[\mathrm{Fe} / \mathrm{H}]<-1$ can be calculated very simply from the observed $\mathrm{Eu}$ and $\mathrm{Fe}$ abundances. Whether this model is a reasonable physical description of Galactic chemical evolution for $r$-elements or is a parametric description with alternative explanations cannot be answered at present.

We wish to thank the reviewers Achim Weiss and an unidentified person for their comments and for suggesting a more extensive treatment of the model. In particular, Achim Weiss has raised several issues requiring discussion. A new data set that appeared after the original submission and two additional data sets are now treated. We have profited greatly from comments and continued enthusiastic support by Roger Blandford and the interest of Marc Kamionkowski. Maurizio Busso has generously given aid and advice on the s-process problems considered here. A conversation with Wal Sargent stimulated our thinking about the mixing problem. This work was supported in part by DOE grants DE-FG02-87ER40328 and DE-FG02-00ER41149 (Y.Z. Q.) and by NASA grant NAG5-4083 (G. J. W.), Caltech Division Contribution 8741(1072).

\section{REFERENCES}

Anders, E., \& Grevesse, N. 1989, Geochim. Cosmochim. Acta, 53, 197

Aoki, W., Norris, J. E., Ryan, S. G., Beers, T. C., \& Ando, H. 2000, ApJ, 536, L97

Arlandini, C., Käppler, F., Wisshak, K., Gallino, R., Lugaro, M., Busso, M., \& Straniero, O. 1999, ApJ, 525, 886

Baraffe, I., Heger, A., \& Woosley, S. E. 2001, ApJ, 550, 890

Brazzle, R. H., Pravdivtseva, O. V., Meshik, A. P., \& Hohenberg, C. M. 1999, Geochim. Cosmochim. Acta, 63, 739

Bromm, V., Coppi, P. S., \& Larson, R. B. 1999, ApJ, 527, L5

Bromm, V., Ferrara, A., Coppi, P. S., \& Larson, R. B. 2001, MNRAS, submitted

Burbidge, E. M., Burbidge, G. R., Fowler, W. A., \& Hoyle, F. 1957, Rev. Mod. Phys., 29, 547

Burris, D. L., Pilachowski, C. A., Armandroff, T. E., Sneden, C., Cowan, J. J., \& Roe, H. 2000, ApJ, 544, 302

Busso, M., Gallino, R., \& Wasserburg, G. J. 1999, ARA\&A, 37, 239

Cameron, A. G. W. 1957, PASP, 69, 201

Cayrel, R., et al. 2001, Nature, 409, 691

Ezer, D., \& Cameron, A. G. W. 1971, Ap\&SS, 14, 399

Freiburghaus, C., Rembges, J.-F., Rauscher, T., Kolbe, E., Thielemann,

F.-K., Kratz, K.-L., Pfeiffer, B., \& Cowan, J. J. 1999, ApJ, 516, 381
Fryer, C. L., Woosley, S. E., \& Heger, A. 2001, ApJ, 550, 372

Gallino, R., Arlandini, C., Busso, M., Lugaro, M., Travaglio, C., Straniero, O., Chieffi, A., \& Limongi, M. 1998, ApJ, 497, 388

Gratton, R. G., \& Sneden, C. 1994, A\&A, 287, 927

Heger, A., Baraffe, I., Fryer, C. L., \& Woosley, S. E. 2000 (astro-ph/ $0010206)$

Hill, V., Plez, B., Cayrel, R., \& Beers, T. C. 2001 (astro-ph/0104172)

Hoffman, R. D., Woosley, S. E., \& Qian, Y.-Z. 1997, ApJ, 482, 951

Johnson, J. A., \& Bolte, M. 2001, ApJ, 554, 888

Käppler, F., Beer, H., \& Wisshak, K. 1989, Rep. Prog. Phys., 52, 945

Kratz, K.-L., Bitouzet, J.-P., Thielemann, F.-K., Möller, P., \& Pfeiffer, B. 1993, ApJ, 403, 216

Magain, P. 1989, A\&A, 209, 211

McWilliam, A. 1998, AJ, 115, 1640

McWilliam, A., Preston, G. W., Sneden, C., \& Searle, L. 1995, AJ, 109, 2757

Meyer, B. S., \& Brown, J. S. 1997, ApJS, 112, 199

Preston, G. W., \& Sneden, C. 2001, ApJ, submitted

Qian, Y.-Z. 2000, ApJ, 534, L67

.2001, ApJ, 552, L117

Qian, Y.-Z., Vogel, P., \& Wasserburg, G. J. 1998, ApJ, 494, 285

Qian, Y.-Z., \& Wasserburg, G. J. 2000, Phys. Rep., 333, 77 
Qian, Y.-Z., \& Wasserburg, G. J. 2001a, ApJ, 549, 337 2001b, ApJ, 552, L55 (QW01)

Raiteri, C. M., Villata, M., Gallino, R., Busso, M., \& Cravanzola, A. 1999, ApJ, 518, L91

Ryan, S. G., Norris, J. E., \& Beers, T. C. 1996, ApJ, 471, 254

Sneden, C., Cowan, J. J., Ivans, I. I., Fuller, G. M., Burles, S., Beers, T. C., \& Lawler, J. E. 2000, ApJ, 533, L139

Sneden, C., McWilliam, A., Preston, G. W., Cowan, J. J., Burris, D. L., \& Armosky, B. J. 1996, ApJ, 467, 819

Thornton, K., Gaudlitz, M., Janka, H.-Th., \& Steinmetz, M. 1998, ApJ, 500,95

Note added in proof.-A subsequent paper (Y.-Z. Qian \& G. T. Wasseburg 2001, in preparation) extends the approach used here to treat almost all the elements between $\mathrm{O}$ and Sr. This complements the present work but follows a different interpretation of the prompt inventory by including concomitant contributions from $H$ and $L$ events in addition to the major contributions from very massive stars. The results of the new paper do not affect the discussion of $r$-process elements presented here for $-3 \lesssim[\mathrm{Fe} / \mathrm{H}]<-1$.
Timmes, F. X., Woosley, S. E., \& Weaver, T. A. 1995, ApJS, 98, 617

Travaglio, C., Galli, D., Gallino, R., Busso, M., Ferrini, F., \& Straniero, O. 1999, ApJ, 521, 691

Truran, J. W. 1981, A\&A, 97, 391

Wasserburg, G. J., Busso, M., \& Gallino, R. 1996, ApJ, 466, L109 (WBG96)

Wasserburg, G. J., \& Qian, Y.-Z. 2000, ApJ, 529, L21 (WQ00)

Westin, J., Sneden, C., Gustafsson, B., \& Cowan, J. J. 2000, ApJ, 530, 783

Woosley, S. E., \& Hoffman, R. D. 1992, ApJ, 395, 202

Zhao, G., \& Magain, P. 1991, A\&A, 244, 425 